Antofagasta - Chile

\title{
ON A SYSTEM OF EVOLUTION EQUATIONS OF MAGNETOHYDRODYNAMIC TYPE: AN ITERATIONAL APPROACH *
}

\author{
E. A. NOTTE-CUELLO ${ }^{\dagger}$ \\ Universidad de Antofagasta, Chile. \\ and \\ M. A. ROJAS-MEDAR ‡ \\ DMA-IMECC-UNICAMP, Brasil.
}

\begin{abstract}
In this work we present a new proof of the existence and uniqueness of strong solution for the magnetohydrodynamic type equations. We use an iterational approach and we give the convergence-rates for this method.
\end{abstract}

*Partially supported by research grant 1977/3711, FAPESP-Brazil.

${ }^{\dagger}$ Partially supported by research grant 98/00619-9, FAPESP-Brazil.

${ }^{\ddagger}$ Partially supported by research grant 300116/93(RN), CNPq. 


\section{Introduction}

In several situations the motion of incompressible electrical conducting fluid can be modelled by the magnetohydrodynamics equation, which correspond to the Navier-Stokes equations coupled with the Maxwell equations. In presence of a free motion of heaby ions, not directly due to the electrical field (see Schlüter [11], and Pikelner [8]), the magnetohydrodynamics equation can be reduced to

$$
\begin{gathered}
\frac{\partial u}{\partial t}-\frac{\eta}{\rho_{m}} \Delta u+u \cdot \nabla u-\frac{\mu}{\rho_{m}} h \cdot \nabla h=f-\frac{1}{\rho_{m}} \nabla\left(p^{*}+\frac{\mu}{2} h^{2}\right) \\
\frac{\partial h}{\partial t}-\frac{1}{\mu \sigma} \Delta h+u \cdot \nabla h-\nabla u= \\
\operatorname{div} u=0 \\
\operatorname{div} h=0
\end{gathered}
$$

together with suitable boundary and initial conditions.

Here, $u$ and $h$ are respectively the unknown velocity and magnetic fields; $p^{*}$ is the unknown hydrostatic pressure; $w$ is an unknown function related to the motion of heavy ions (in such way that the density of electric current, $j_{0}$, generated by this motion satisfies the relation $\left.\operatorname{rot} j_{0}=-\sigma \nabla w\right) ; \rho_{m}$ is the density of mass of the fluid (assumed to be a positive constant); $\mu>0$ is the constant magnetic permeability of the medium; $\sigma>0$ is the constant electric conductivity; $\eta>0$ is the constant viscosity of the fluid; $f$ is an given external force field.

We append to equation (1.1) the following initial and boundary conditions

$$
\left.u\right|_{\partial \Omega}=0,\left.\quad h\right|_{\partial \Omega}=0
$$

$$
u(0)=u_{0}, \quad h(0)=h_{0} \quad \text { in } \quad \Omega .
$$

where $u_{0}$ and $h_{0}$ are given functions.

In this paper, we consider the approximation of the problem (1.1)-(1.2) by an iterative process. We prove that the sequence build by this method converge for a unique strong solution of (1.1)-(1.2). We also give the correspondent convergence rates in several norms.

Let us cite the classical works on the initial value problem (1.1)-(1.2) and locate our contribution therein.

Lassner [6], Fujita and Kato [5] show the local existence and uniqueness of strong solutions by using semigroup techniques. A more constructive 
spectral Galerkin method was performed by Boldrini and Rojas-Medar [2], [9]. In their paper, one obtains a local global existence and uniqueness of strong solutions, but they not develop the convergence rates. The eigenfunctions of the associated Stokes operador are used as a basis of approximation. Since by working with arbitrary domains, it is complicated to write down this basis, the study of other methods of approximation becomes very important. For another related works, we refer the reader to [9], [4].

The study of the Fitz-Hug problem via an iterative method is presented in the work of Sedov and Fokht [12]. In such a paper they prove the convergence rates. Following Sedov and Fokht, Zarubin [14] proposed an iterative method for finding the approximate solution of an initial and boundary value problem for the heat-convection equation at level of Boussinesq approximation. The statement of Theorem 1, p. 1081 in [14] furnishes a convergence ratc, the proof of this result is incorrect.

In this paper, we use this type of method to prove global existence and uniqueness of strong solution for the two and three-dimensional magnetohydrodynamic type equations. We also give the convergence rates of this method in several norms.

We observe that our arguments are also true in the problem studied by Zarubin.

The paper is organized as follows.

In Section 2 we state the basic assumptions and results which we will use in the paper. In the Section 3, we prove estimates for the sequence $\left(u^{n}, h^{n}\right)$. In the Section 4, we prove several convergence-rates bounds for the approximate solutions. Finally, in the Section 5, we obtain some results on the pressures.

\section{Preliminaries}

Let $\Omega$ be a bounded domain in $\mathbf{R}^{N}, N=2$ or 3 , with a smooth boundary $\partial \Omega, T>0$ an arbitrary number finite. The functions in this paper are either $\mathbf{R}$ or $\mathbf{R}^{N}$-valued, and sometimes we will not distinguish them in our notations, when it will be clear from the context. The $L^{2}$-norm will be denoted by $\|$.$\| and (.,$.$) the L^{2}$-inner product. We consider the usual Sobolev spaces

$$
W^{m, q}(D)=\left\{f \in L^{q}(D)\left|\left\|\partial^{\alpha} f\right\|_{L^{q}(D)}<\infty,\right| \alpha \mid \leq m\right\}
$$

for $m \in N, 1 \leq q \leq \infty, D=\Omega$ or $D=\Omega \times(0, T), 0<T<\infty$, with the usual norm. When $q=2$, we denote $H^{m}(D)=W^{m, 2}(D)$ and $H_{0}^{m}(D)=$ 
closure of $C_{0}^{\infty}(D)$ in $H^{m}(D)$.

We put.

$$
\begin{aligned}
& C_{0, \sigma}^{\infty}(\Omega)=\left\{v \in C_{0}^{\infty}(\Omega) / \text { div }=0\right\} \\
& H=\text { closure of } C_{0, \sigma}^{\infty}(\Omega) \text { in } L^{2}(\Omega), \\
& V=\text { closure of } C_{0, \sigma}^{\infty}(\Omega) \text { in } H^{1}(\Omega),
\end{aligned}
$$

It is possible to show that

$$
V=\left\{v \in H_{0}^{1}(\Omega) / \operatorname{div} v=0\right\} .
$$

We recall the Helmholtz decomposition of vector field: $L^{2}(\Omega)=H \oplus G$, being $G=\left\{\phi \mid \phi=\nabla p, p \in H^{1}(\Omega)\right\}$.

Throughout the paper, $P$ will denote the orthogonal projection from $L^{2}(\Omega)$ onto $H$. The operator $A: D(A) \subset H \longrightarrow H$, given by $A=-P \Delta$ with domain $D(A)=V \cap H^{2}(\Omega)$ is called the Stokes operator. It is well known that $A$ is a positive definite self-adjoint operator and it is characterized by the relation

$$
(A w, v)=(\nabla w, \nabla v) \text { for all } w \in D(A), v \in V .
$$

We observe that when $\Omega$ is of class $C^{1,1}$, we have that the norms $\|u\|_{H^{2}}$ and $\|A u\|$ are equivalents (see Anrouche and Girault [1]). The properties of $A$ are known, see Constantin and Foias [3], Lions [7] or Temam [13].

By using the properties of $P$, we can reformulate the problem (1.1)(1.2), in the following way. Let us find $u, h$ in suitable spaces, these space will be exactly defined later, which satisfy

$$
\begin{aligned}
& \alpha \frac{d u}{d t}+\alpha P(u . \nabla u)-P(h . \nabla h)+\nu A u=\alpha f, \\
& \frac{d h}{d t}+P(u . \nabla h)-P(h . \nabla u)+\gamma A h=0 \\
& \text { for } \quad 0<t<T \\
& u(0)=u_{0}, \quad h(0)=h_{0}
\end{aligned}
$$

Where, $\alpha=\frac{\rho_{m}}{\mu}, \nu=\frac{\eta}{\mu}, \quad \gamma=\frac{1}{\mu \sigma}$.

We now define strong solutions of the problem (2.1).

\section{Definition 2.1 :}

Let $u_{0}, h_{0} \in V$ and $f \in L^{2}\left(0, T ; L^{2}(\Omega)\right)$. By a strong solution of the problem (2.1), we mean a pair of vector-valued functions $(u, h)$ such that 


$$
u, h \in L^{\infty}(0, T ; V) \cap L^{2}(0, T ; D(A))
$$

and that satisfies (2.1)

\section{Remark 2.2 :}

In what follows, we will prove that if $(u, h)$ is a strong solution of (2.1) then $u_{t}, h_{t} \in L^{2}(0, T ; H)$. This condition, together with $u, h \in$ $L^{2}(0, T ; D(A))$, implies by interpolation (see, Temam [13], p. 260), that $u, h$ are almost everywhere equal to a continuous functions from $[0, T]$ into $V$. Consequently, the initial condition $u(0)=u_{0}$ and $h(0)=h_{0}$ are meaningful.

We consider the following iterative process for the approximate solution of problem (2.1), we consider

$$
u^{1}(t)=e^{A t} u_{0} \quad \text { and } \quad h^{1}(t)=e^{A t} h_{0} .
$$

For given $u^{n}$ and $h^{n}$, we define $u^{n+1}$ and $h^{n+1}$ by

$$
\begin{aligned}
& \alpha \frac{d u^{n+1}}{d t}+\nu A u^{n+1}+P\left(u^{n} \cdot \nabla u^{n+1}\right)-P\left(h^{n} \cdot \nabla h^{n+1}\right)=P(\alpha f) \\
& \frac{d h^{n+1}}{d t}+\gamma A h^{n+1}+P\left(u^{n} \cdot \nabla h^{n+1}\right)-P\left(h^{n} \cdot \nabla u^{n+1}\right)=0 \\
& u^{n+1}(0)=u_{0} \quad h^{n+1}(0)=h_{0} .
\end{aligned}
$$

In the following sections, we justify this procedure by making use of the energy method.

\section{Existence of unique solution of problem (2.2)}

To see that the system (2.2) has an unique solution we can used, for example, the Galerkin method as in Boldrini and Rojas-Medar [2], [10] or, the semigroup approach as in Fujita and Kato [5]. In fact, let $V_{k}=$ $\left\langle w^{1}(x), \ldots, w^{k}(x)\right\rangle$ by the subspace of $V \cap H^{2}(\Omega)$ spanned by the $k$-first eigenfunctions of the Stokes operator, we put

$$
\begin{aligned}
& u_{k}^{n+1}(t)=\sum_{i=1}^{k} C_{i n}^{k}(t) w^{i}(x) \\
& h_{k}^{n+1}(t)=\sum_{i=1}^{k} C_{i n}^{k}(t) w^{i}(x)
\end{aligned}
$$


solution of

$$
\begin{aligned}
& \alpha \frac{d u_{k}^{n+1}}{d t}+\nu A u_{k}^{n+1}=P_{k}(\alpha f)-P_{k}\left(u_{k}^{n} \cdot \nabla u_{k}^{n+1}\right)+P\left(h_{k}^{n} \cdot \nabla h_{k}^{n+1}\right) \\
& \frac{d h_{k}^{n+1}}{d t}+\gamma A h_{k}^{n+1}=P_{k}\left(h_{k}^{n} \cdot \nabla u_{k}^{n+1}\right)-P_{k}\left(u_{k}^{n} \cdot \nabla h_{k}^{n+1}\right) \\
& u_{k}^{n+1}(0)=P_{k} u_{0}, \quad h_{k}^{n+1}(0)=P_{k} h_{0}
\end{aligned}
$$

As in Boldrini and Rojas-Medar [2], it can be proved that the system (2.3) has an unique solution $\left(u_{k}^{n+1}, h_{k}^{n+1}\right)$ in an interval $[0, T]$. We give the precise results to future reference.

\section{Lemma 2.3 :}

If $f \in L^{2}\left(0, T ; L^{2}(\Omega)\right), u_{0}, h_{0} \in V$ then

$$
\begin{aligned}
& u^{n+1}, h^{n+1} \in L^{\infty}(0, T ; V) \cap L^{2}(0, T ; D(A)) \\
& u_{t}^{n+1}, h_{t}^{n+1} \in L^{2}(0, T ; H) .
\end{aligned}
$$

Moreover, $u^{n+1}, h^{n+1} \in C([0, T] ; V)$.

\section{Lemma 2.4 :}

If $f, f_{t} \in L^{2}\left(0, T ; L^{2}(\Omega)\right), u_{0}, h_{0} \in D(A)$, then

$$
\begin{aligned}
& u^{n+1}, h^{n+1} \in L^{\infty}(0, T ; D(A)), \\
& u_{t}^{n+1}, h_{t}^{n+1} \in L^{\infty}(0, T ; H) \cap L^{2}(0, T ; V), \\
& u_{t t}^{n+1}, h_{t t}^{n+1} \in L^{2}\left(0, T ; V^{*}\right) .
\end{aligned}
$$

Therefore, $u^{n+1}, h^{n+1} \in C^{1}([0, T] ; H) \cap C([0, T] ; D(A))$.

By using the Amrouche-Girault results [1], we can obtain the following corollary.

\section{Corollary 2.5 :}

Under the conditions of the Lemma 2.3, there exists unique functions

$$
p^{n+1}, w^{n+1} \in L^{2}\left(0, T ; H^{1}(\Omega) / \mathbb{R}\right)
$$

such that $\left(u^{n+1}, h^{n+1}, p^{n+1}, w^{n+1}\right)$ is a solution of problem 


$$
\frac{\partial u^{n+1}}{\partial t}-\frac{\eta}{\rho_{m}} \Delta u^{n+1}+u^{n} \cdot \nabla u^{n+1}-\frac{\mu}{\rho_{m}} h^{n} \cdot \nabla h=\frac{1}{\rho_{m}} \nabla p^{n+1},
$$

$$
\begin{gathered}
\frac{\partial h^{n+1}}{\partial t}-\frac{1}{\mu \sigma} \Delta h^{n+1}+u^{n} \cdot \nabla h^{n+1}-h^{n} \cdot \nabla u^{n+1}=-\operatorname{gradw} w^{n+1} \\
\operatorname{div} u^{n+1}=0 \\
\operatorname{div} h^{n+1}=0
\end{gathered}
$$

$$
\begin{aligned}
& \left.u^{n+1}\right|_{\partial \Omega}=0,\left.\quad h^{n+1}\right|_{\partial \Omega}=0, \\
& u^{n+1}(0)=u_{0}, \quad h^{n+1}(0)=h_{0} \quad \text { in } \quad \Omega,
\end{aligned}
$$

where $p^{n+1}=\left(p^{*}\right)^{n+1}+\frac{\mu}{2}\left(h^{n+1}\right)^{2}$.

If the hypotheses of the Lemma 2.4 are verified, then

$$
p^{n+1}, w^{n+1} \in L^{\infty}\left(0, T ; H^{1}(\Omega) / \mathbb{I R}\right) .
$$

\section{A Priori Estimates}

In this section, we prove several estimates for the sequence $\left(u^{n}, h^{n}\right)$. In the following, we assume $u_{0}=h_{0}=0$ for simplicity of exposition. We have:

\section{Lemma 3.1 :}

If $f \in L^{2}\left(0, T ; L^{2}(\Omega)\right)$, then the approximate solutions $\left(u^{n}, h^{n}\right)$ given by the iterative process (2.3), satisfy uniformly in $n$ the following estimates

$$
\begin{gathered}
\sup _{t}\left\|u^{n}(t)\right\| \leq I_{0}, \quad \sup _{t}\left\|h^{n}(t)\right\| \leq \alpha^{1 / 2} M_{0}, \\
\left\|u^{n}\right\|_{L^{2}(0, T ; V)} \leq\left(\frac{\alpha}{\nu}\right)^{1 / 2} M_{0},\left\|h^{n}\right\|_{L^{2}(0, T ; V)} \leq\left(\frac{\alpha}{2 \gamma}\right)^{1 / 2} M_{0}, \\
\text { where } \quad M_{0}=\left(\frac{\alpha}{2 \gamma \lambda}\right)^{1 / 2}\|f\|_{L^{2}(Q)} \quad \text { and }
\end{gathered}
$$

$\lambda$ is the smallest eigenvalue of the operator $-\Delta$ subject to zero boundary condition.

Proof. : 
By multiplying $(2.3)_{i}$ by $u^{n+1}$ and $(2.3)_{i i}$ by $h^{n+1}$, we obtain

$$
\begin{aligned}
& \frac{\alpha}{2} \frac{d}{d t}\left\|u^{n+1}\right\|^{2}+\nu\left\|\nabla u^{n+1}\right\|^{2}=\left(\alpha f, u^{n+1}\right)+\left(h^{n} . \nabla h^{n+1}, u^{n+1}\right) \\
& \frac{1}{2} \frac{d}{d t}\left\|h^{n+1}\right\|^{2}+\gamma\left\|\nabla h^{n+1}\right\|^{2}=+\left(h^{n} \cdot \nabla u^{n+1}, h^{n+1}\right) .
\end{aligned}
$$

By observing that $\left(h^{n} \cdot \nabla h^{n+1}, u^{n+1}\right)+\left(h^{n} \cdot \nabla u^{n+1}, h^{n+1}\right)=0$, and using the above equalities, we find

$$
\frac{1}{2} \frac{d}{d t}\left(\alpha\left\|u^{n+1}\right\|^{2}+\left\|h^{n+1}\right\|^{2}\right)+\nu\left\|\nabla u^{n+1}\right\|^{2}+\gamma\left\|\nabla h^{n+1}\right\|^{2}=\left(\alpha f, u^{n+1}\right) .
$$

Now, by integrating the above equality with respect to $t$, where $0 \leq t \leq T$, we get

$$
\begin{aligned}
& \frac{\alpha}{2}\left\|u^{n+1}\right\|^{2}+\frac{1}{2}\left\|h^{n+1}\right\|^{2}+\int_{0}^{t} \nu\left\|\nabla u^{n+1}\right\|^{2} d s+\gamma \int_{0}^{t}\left\|\nabla h^{n+1}\right\|^{2} d s \\
\leq & \int_{0}^{t}\left(\alpha f(s), u^{n+1}(s)\right) d s \\
\leq & \frac{\alpha^{2}}{2 \varepsilon}\|f\|_{L^{2}(Q)}^{2}+\frac{\varepsilon}{2 \lambda} \int_{0}^{t}\left\|\nabla u^{n+1}\right\|^{2} d s,
\end{aligned}
$$

where $\lambda$ is smallest eigenvalue of the operator $-\Delta$ subject to zero boundary condition.

By putting $\varepsilon=\nu \lambda$, we obtain

$$
\begin{aligned}
& \alpha\left\|u^{n+1}(t)\right\|^{2}+\left\|h^{n+1}(t)\right\|^{2}+\nu \int_{0}^{t}\left\|\nabla u^{n+1}(s)\right\|^{2} d s \\
& +2 \gamma \int_{0}^{t}\left\|\nabla h^{n+1}(s)\right\|^{2} d s \leq \frac{\alpha^{2}}{\nu \lambda}\|f\|_{L^{2}(Q)}^{2},
\end{aligned}
$$

Consequently,

$$
\begin{aligned}
& \sup _{t}\left\|u^{n+1}(t)\right\|^{2} \leq\left(\frac{\alpha}{2 \nu \lambda}\right)^{1 / 2}\|f\|_{L^{2}(Q)} \equiv M_{0}, \\
& \sup _{t}\left\|h^{n+1}(t)\right\| \leq M_{0} \alpha^{1 / 2} \\
& \left\|u^{n+1}\right\|_{L^{2}(0, T ; V)} \leq\left(\frac{\alpha}{\nu}\right)^{1 / 2} M_{0} \\
& \left\|h^{n+1}\right\|_{L^{2}(0, T ; V)} \leq\left(\frac{\alpha}{2 \gamma}\right)^{1 / 2} M_{0} .
\end{aligned}
$$




\section{Lemma 3.2 :}

If $f \in L^{2}\left(0, T ; L^{2}(\Omega)\right)$ and $N=2$, then the approximate solutions $\left(u^{n}, h^{n}\right)$ given by the iterative process $(2.2)$, satisfy uniformly in $n$ the following estimates

$$
\begin{aligned}
& \sup _{t}\left\|\nabla u^{n}(t)\right\| \leq M_{1}, \quad \sup _{t}\left\|\nabla h^{n}(t)\right\| \leq \alpha^{1 / 2} M_{1}, \\
& \text { where } \quad M_{1}=\left(\frac{C}{\alpha}\right)^{1 / 2}\|f\|_{L^{2}(Q)}\left[\exp \left(\frac{\alpha}{\nu}\right)^{1 / 2} M_{0}\right]^{1 / 2}
\end{aligned}
$$

The estimate (3.3) is true in the three-dimensional case if

$$
\left(\nu-\lambda^{-1 / 8} C_{\Omega} M M_{1}\left(2^{3 / 4}+4\right)\right)>0 \text { and }\left(2 \gamma-\lambda^{-1 \backslash 8} C_{\Omega} M M_{1}\left(2^{2 / 3}+2^{5 / 3}\right)\right)>0
$$

where $\lambda$ is the smallest eigenvalue of the Laplace operator subject to the Dirichlet condition, $\lambda>0$ and $M$ is the Cattabriga's constant.

\section{Proof. :}

By multiplying $(2.2)_{i}$ by $A u^{n+1},(2.2)_{i i}$ by $A h^{n+1}$, and integrating over $\Omega$, we get

$$
\begin{aligned}
& \frac{\alpha}{2} \frac{d}{d t}\left\|\nabla u^{n+1}\right\|^{2}+\nu\left\|A u^{n+1}\right\|^{2} \\
& \quad=\left(\alpha f, A u^{n+1}\right)-\left(u^{n} \cdot \nabla u^{n+1}, A u^{n+1}\right)+\left(h^{n} \cdot \nabla h^{n+1}, A u^{n+1}\right),
\end{aligned}
$$

$$
\begin{aligned}
& \frac{1}{2} \frac{d}{d t}\left\|\nabla h^{n+1}\right\|^{2}+\gamma\left\|A h^{n+1}\right\|^{2} \\
& \quad=\left(h^{n} \cdot \nabla u^{n+1}, A h^{n+1}\right)-\left(u^{n} \cdot \nabla h^{n+1}, A h^{n+1}\right) .
\end{aligned}
$$

Let us prove the estimate (3.3) for the case $n=2$. To cloing it, we recall the following inequality given, for example in [[13], pp. 291]

$$
\|\phi\|_{L^{4}} \leq C\|\phi\|^{1 / 2}\|\nabla \phi\|^{1 / 2}
$$


We use the previous inequality to prove that,

$$
\begin{aligned}
(3.6)|(w, \nabla \varphi, A \psi)| & \leq\|w\|_{L^{4}}\|\nabla \varphi\|_{L^{4}}\|A \psi\| \\
& \leq \alpha\|w\|^{1 / 2}\|\nabla w\|^{1 / 2}\|\nabla \varphi\|^{1 / 2}\|\nabla(\nabla \varphi)\|^{1 / 2}\|A \psi\| \\
& \leq C_{\varepsilon}\|w\|\|\nabla w\|\|\nabla \varphi\|\|A \varphi\|+\varepsilon\|A \psi\|^{2} \\
& \leq C_{\varepsilon, \delta}\|w\|^{2}\|\nabla w\|^{2}\|\nabla \varphi\|^{2}+\delta\|A \varphi\|^{2}+\varepsilon\|A \psi\|^{2}
\end{aligned}
$$

In the same way, we find

$$
|(w . \nabla \varphi, A \varphi)| \leq C_{\varepsilon}\|w\|^{2}\|\nabla \varphi\|^{2}\|\nabla w\|^{2}+\varepsilon\|A \varphi\|^{2} .
$$

By using the estimate (3.7) in the second term of right-hand of the equality $(3.4)_{i}$ and (3.7) for the second term of right-hand of $(3.4)_{i i}$, we obtain

$$
\begin{aligned}
& \left|\left(u^{n} . \nabla u^{n+1}, A u^{n+1}\right)\right| \leq C_{\varepsilon}\left\|u^{n}\right\|^{2}\left\|\nabla u^{n+1}\right\|^{2}\left\|\nabla u^{n}\right\|^{2}+\varepsilon\left\|A u^{n+1}\right\|^{2}, \\
& \left|\left(u^{n} \cdot \nabla h^{n+1}, A h^{n+1}\right)\right| \leq C_{\delta}\left\|u^{n}\right\|^{2}\left\|\nabla h^{n+1}\right\|^{2}\left\|\nabla u^{n}\right\|^{2}+\delta\left\|A h^{n+1}\right\|^{2} .
\end{aligned}
$$

By using the estimate (3.6) for the third term in the right-hand of the equality $(3.4)_{i}$ and for the first term in the right-hand of the equality (3.4) ${ }_{i i}$, we have

$$
\begin{aligned}
& \left|\left(h^{n} . \nabla h^{n+1}, A u^{n+1}\right)\right| \leq C_{\varepsilon, \delta}\left\|h^{n}\right\|^{2}\left\|\nabla h^{n}\right\|^{2}\left\|\nabla h^{n+1}\right\|^{2}+\delta\left\|A h^{n+1}\right\|^{2}+ \\
& \quad+\varepsilon\left\|A u^{n+1}\right\|^{2}, \\
& \left|\left(h^{n} \cdot \nabla u^{n+1}, A h^{n+1}\right)\right| \leq C_{\varepsilon, \delta}\left\|h^{n}\right\|^{2}\left\|\nabla h^{n}\right\|^{2}\left\|\nabla u^{n+1}\right\|^{2}+\delta\left\|A h^{n+1}\right\|^{2}+ \\
& \quad+\varepsilon\left\|A u^{n+1}\right\|^{2} .
\end{aligned}
$$

The Cauchy-Schwarz and Young inequalities imply

$$
\left|\left(\alpha f, A u^{n+1}\right)\right| \leq C_{\varepsilon}\|f\|^{2}+\varepsilon\left\|A u^{n+1}\right\|^{2} .
$$

By adding the equalities (3.4) $i$ and (3.4) $)_{i i}$, and using the previous estimates, with $\varepsilon=\frac{\nu}{8}, \delta=\frac{\gamma}{6}$, and the estimate (3.1), we have

$$
\begin{gathered}
\frac{d}{d t}\left(\alpha\left\|\nabla u^{n+1}\right\|^{2}+\left\|\nabla h^{n+1}\right\|^{2}\right)+\nu\left\|A u^{n+1}\right\|+\gamma\left\|A h^{n+1}\right\| \\
\leq C\|f\|^{2}+C\left(\left\|\nabla h^{n}\right\|^{2}+\left\|\nabla u^{n}\right\|^{2}\right)\left(\left\|\nabla h^{n+1}\right\|^{2}+\alpha\left\|\nabla u^{n+1}\right\|^{2}\right) .
\end{gathered}
$$

By integrating with respect to $t$ the last inequality, we get $\alpha\left\|\nabla u^{n+1}(t)\right\|^{2}+\left\|\nabla h^{n+1}(t)\right\|^{2}+\nu \int_{0}^{t}\left\|A u^{n+1}(s)\right\|^{2} d s+\gamma \int_{0}^{l}\left\|A h^{n+1}(s)\right\|^{2} d s$ 


$$
\begin{aligned}
& \leq C\|f\|_{L^{2}(Q)}^{2}+C \int_{0}^{t}\left(\left\|\nabla h^{n}(s)\right\|^{2}+\left\|\nabla u^{n}(s)\right\|^{2}\right)\left(\left\|\nabla h^{n+1}(s)\right\|^{2}+\right. \\
& \left.\quad+\alpha\left\|\nabla u^{n+1}(s)\right\|^{2}\right) d s .
\end{aligned}
$$

By using the Gronwall inequality, the last relation yields

$$
\begin{gathered}
\alpha\|\nabla u(t)\|+\|\nabla h(t)\|+\nu \int_{0}^{t}\|A u(s)\| d s+\gamma \int_{0}^{t}\|A h(s)\| d s \\
\leq C\|f\|_{L^{2}(Q)}^{2} \exp \left(\int_{0}^{t}\left(\left\|\nabla u^{n}(s)\right\|^{2}+\left\|\nabla h^{n}(s)\right\|^{2}\right) d s\right. \\
\leq C\|f\|_{L^{2}(Q)}^{2} \exp \left(\left(\frac{\alpha}{\gamma}\right)^{1 / 2} M_{0}\right)
\end{gathered}
$$

where we used the estimates (??).

To prove the estimate (3.3) in the three-dimensional case, we recall the following inequality [[13], pp.296].

$$
\|v\|_{L^{4}} \leq 2^{1 / 2}\|v\|^{1 / 4}\|\nabla v\|^{3 / 4} .
$$

We use this inequality to prove the following estimates

$$
\begin{aligned}
\left|\left(u^{n} \cdot \nabla u^{n+1}, A u^{n+1}\right)\right| & \leq\left\|u^{n}\right\|_{L^{4}}\left\|\nabla u^{n+1}\right\|_{L^{4}}\left\|A u^{n+1}\right\| \\
& \leq 2^{1 / 2}\left\|u^{n}\right\|^{1 / 4}\left\|\nabla u^{n}\right\|^{3 / 4}\left\|\nabla u^{n+1}\right\|_{L^{4}}\left\|A u^{n+1}\right\| \\
& \leq 2^{1 / 2}\left\|u^{n}\right\|^{1 / 4}\left\|\nabla u^{n}\right\|^{3 / 4} C_{\Omega} M\left\|A u^{n+1}\right\|^{2} .
\end{aligned}
$$

The inequality $\|v\| \leq \frac{1}{\sqrt{\lambda}}\|\nabla v\|$, we get

$$
\left|\left(u^{n} \cdot \nabla u^{n+1}, A u^{n+1}\right)\right| \leq 2^{1 / 2} \lambda^{-1 / 8}|| \nabla u^{n}|| C_{\Omega} \bar{M}\left\|A u^{n+1}\right\|^{2} .
$$

Analogously,

$$
\begin{aligned}
&\left|\left(u^{n} \cdot \nabla h^{n+1}, A h^{n+1}\right)\right| \leq 2^{1 / 2} \lambda^{-1 / 8} C_{\Omega} \bar{M}|| \nabla u^{n}|||| A h^{n+1} \|^{2} \\
&\left|\left(h^{n} \cdot \nabla h^{n+1}, A u^{n+1}\right)\right| \leq 2^{1 / 2} \lambda^{-1 / 8} C_{\Omega} \bar{M}|| \nabla h^{n}|||| A h^{n+1}|||| A u^{n+1} \| \\
&\left|\left(h^{n} \cdot \nabla h^{n+1}, A h^{n+1}\right)\right| \leq 2^{1 / 2} \lambda^{-1 / 8} C_{\Omega} \bar{M}|| \nabla h^{n}|||| A u^{n+1}|||| A h^{n+1} \| .
\end{aligned}
$$

By using the Cauchy-Schwarz and Young inequalities, we obtain

$$
\left|\left(\alpha f, A u^{n+1}\right)\right| \leq \frac{\alpha^{2}}{\nu}\|f\|^{2}+\frac{\nu}{2}\left\|A u^{n+1}\right\|^{2} .
$$

The previous estimates, gives 
$\frac{d}{d t}\left(\alpha\left\|\nabla u^{n+1}\right\|^{2}+\left\|\nabla h^{n+1}\right\|^{2}\right)+$

$+\left(\nu-2 \cdot 2^{1 / 2} \lambda^{-1 / 8} C_{\Omega} \bar{M}\left\|\nabla u^{n}\right\|-2 \cdot 2 \lambda^{-1 / 8} C_{\Omega} \bar{M}^{2}\left\|\nabla h^{n}\right\|\right)\left\|A u^{n+1}\right\|^{2}+$

$+\left(2 \gamma-2 \cdot 2^{1 / 2} \lambda^{-1 / 8} C_{\Omega} \bar{M}\left\|\nabla u^{n}\right\|-4 \cdot 2^{1 / 2} \lambda^{1 / 8} C_{\Omega} \bar{M}\left\|\nabla h^{n}\right\|\right)\left\|A h^{n+1}\right\|^{2} \leq$

$\leq C_{\alpha, \nu}\|f\|^{2}$

Integrating with respect to $t$ the last inequality, we get

$$
\begin{aligned}
& \alpha\left\|\nabla u^{n+1}\right\|^{2}+\left\|\nabla h^{n+1}\right\|^{2}+ \\
& +\int_{0}^{t}\left(\nu-2 \cdot 2^{1 / 2} \lambda^{-1 / 8} C_{\Omega} \bar{M}\left\|\nabla u^{n}\right\|-2 \cdot 2 \lambda^{-1 / 8} C_{\Omega} \bar{M}^{2}\left\|\nabla h^{n}\right\|\right) \\
(3.9) \quad & \left\|A u^{n+1}\right\|^{2} d \tau+ \\
& +\int_{0}^{t}\left(2 \gamma-2 \cdot 2^{1 / 2} \lambda^{1 / 8} C_{\Omega} \overline{M I}\left\|\nabla u^{n}\right\|-4 \cdot 2^{1 / 2} \lambda^{1 / 8} C_{\Omega} \bar{M}\left\|\nabla h^{n}\right\|\right) \\
& \left\|A h^{n+1}\right\|^{2} d \tau \leq C_{\alpha, \nu}\|f\|_{L^{2}(Q) .}^{2}
\end{aligned}
$$

By setting $n=1$ in (3.9) and using the condition of the lemma $\left(u^{1}=h^{1}=0\right)$, we get

$$
\begin{aligned}
& \quad \alpha\left\|\nabla u^{2}\right\|^{2}+\left\|\nabla h^{2}\right\|^{2}+\int_{0}^{t}\left\{\nu\left\|A u^{2}\right\|^{2}+2 \gamma\left\|A h^{2}\right\|^{2}\right\} d \tau \\
& \leq \quad C_{\alpha, \nu}\|f\|_{L^{2}(Q)}^{2}=\bar{N}^{2} .
\end{aligned}
$$

Thus,

(3.10) $\sup _{t \in[0, T]}\left\|\nabla u^{2}(t)\right\|^{2} \leq M_{1}^{2} \quad$ and $\sup _{t \in[0, T]}\left\|\nabla h^{2}(t)\right\|^{2} \leq M_{1}^{2}$

where

$$
M_{1}=\max \left\{\bar{N}^{2}, \frac{\bar{N}^{2}}{\alpha}\right\}
$$


for $n=2$ in (3.9), we obtain

$$
\begin{aligned}
& \alpha\left\|\nabla u^{3}\right\|^{2}+\left\|\nabla h^{3}\right\|^{2} \\
& +\int_{0}^{t}\left(\nu-2^{3 / 2} \lambda^{1 / 8} C_{\Omega} \bar{M}\left\|\nabla u^{2}\right\|-4 \lambda^{1 / 8} C_{\Omega} \bar{M}^{2}\left\|\nabla h^{2}\right\|\right)\left\|A u^{3}\right\|^{2} d \tau \\
& +\int_{0}^{t}\left(2 \gamma-2^{3 / 4} \lambda^{-1 / 8} C_{\Omega} \bar{M}\left\|\nabla u^{2}\right\|-2^{5 / 4} \lambda^{-1 / 8} C_{\Omega} \bar{M}\left\|\nabla h^{2}\right\|\right)\left\|A h^{3}\right\| d \tau \\
& \leq C_{\alpha, \nu}\|f\|_{L^{2}(Q)}^{2}=\bar{N}^{2} .
\end{aligned}
$$

From (3.10), we deduce that $-M_{1} \leq-\left\|\nabla u^{2}(t)\right\|$ and $-M_{1} \leq-\left\|\nabla h^{2}(t)\right\|$, then

$$
\begin{aligned}
& \alpha\left\|\nabla u^{3}\right\|^{2}+\left\|\nabla h^{3}\right\|^{2}+\int_{0}^{t}\left(\nu-\lambda^{-1 / 8} C_{\Omega} \bar{M} M_{1}\left(2^{3 / 4}+4\right)\right)\left\|A u^{3}\right\|^{2} d \tau \\
& +\int_{0}^{t}\left(2 \gamma-\lambda^{-1 / 8} C_{\Omega} \bar{M} M_{1}\left(2^{3 / 2}+2^{5 / 2}\right)\right)\left\|A h^{3}\right\|^{2} d \tau \\
& \leq \bar{N}^{2}
\end{aligned}
$$

Nevertheless, by the hypotheses of lemma, we have

$$
\left(\nu-\lambda^{-1 / 8} C_{\Omega} \bar{M} M_{1}\left(2^{3 / 4}+4\right)\right)>0
$$

and

$$
\left(2 \gamma-\lambda^{-1 / 8} C_{\Omega} \bar{M} M_{1}\left(2^{3 / 2}+2^{5 / 2}\right)\right)>0,
$$

therefore

$$
\sup _{t \in[0, T]}\left\|\nabla u^{3}\right\|^{2} \leq M_{1}^{2} \quad \text { and } \quad \sup _{t \in[0, T]}\left\|\nabla h^{3}\right\|^{2} \leq M_{1}^{2} .
$$

The previous arguments, imply

$$
\sup _{t \in[0, T]}\left\|\nabla u^{n}(t)\right\|^{2} \leq M_{1}^{2} \quad \text { and } \sup _{t \in[0, T]}\left\|\nabla h^{n}\right\|^{2} \leq M_{1}^{2} .
$$

For all $n$, thus

$$
\sup _{t \in[0, T]}\left\|\nabla u^{n}(t)\right\| \leq M_{1} \quad \text { and } \quad \sup _{t \in[0, T]}\left\|\nabla h^{n}(t)\right\| \leq M_{1} .
$$


This completes the proof of the Lemma.

\section{Lemma 3.3 :}

Under the hypotheses of the lemma 3.2 the approximate solutions $\left(u^{n}, h^{n}\right)$ satisfy, uniformly in $n$, the following estimates

$$
\begin{array}{r}
\left\|u^{n}\right\|_{L^{2}(0, T ; D(A))} \leq\left(\frac{C \alpha}{\nu}\right)^{1 / 2} M_{1},\left\|h^{n}\right\|_{L^{2}(0, T ; D(A))} \leq \\
\left(\frac{C \alpha}{\gamma}\right)^{1 / 2} M_{1}
\end{array}
$$

$\left\|u_{t}^{n}\right\|_{L^{2}(0, T ; H)} \leq\left(\frac{C \alpha}{\gamma} M_{1}+C\|f\|_{L^{2}(Q)}^{2}\right)^{1 / 2} ;\left\|h_{t}^{n}\right\|_{L^{2}(0, T ; H)} \leq\left(\frac{C \alpha}{\gamma} M_{1}\right)^{1 / 2}$

$$
\text { where } \quad M_{1}=\left(\frac{C}{\alpha}\right)^{1 / 2}\|f\|_{L^{2}(Q)}\left[\exp \left(\frac{\alpha}{\nu}\right)^{1 / 2} M_{0}\right]^{1 / 2}
$$

\section{Proof :}

We observe that the estimate (??) for the two-dimensional case following from (3.8). In the three-dimensional case, the estimate (??) following from (3.11) and the hypotheses

$$
\left(\nu-\lambda^{-1 / 8} C_{\Omega} M M_{1}\left(2^{3 / 2}+4\right)\right) \leq\left(\nu-\lambda^{-1 / 8} C_{\Omega}\left\|\nabla u^{n}(t)\right\|\left(2^{3 / 2}+2^{5 / 2}\right)\right.
$$

and

$\left(2 \gamma-\lambda^{-1 / 8} C_{\Omega} M M_{1}\left(2^{3 / 2}+2^{5 / 2}\right)\right) \leq\left(2 \gamma-\lambda^{-1 / 8} C_{\Omega} M\left\|\nabla h^{n}(t)\right\|\left(2^{3 / 2}+2^{5 / 2}\right)\right)$

for all $t \in[0, T]$ and for all $n$. Then, (??) implies

$\left(\nu-\lambda^{-1 / 8} C_{\Omega} M M_{1}\left(2^{3 / 2}+4\right) \int_{0}^{T}\left\|A u^{n+1}\right\| d \tau+\left(2^{3 / 2}+2^{5 / 2}\right)\right) \int_{0}^{T}\left\|A h^{n+1}\right\| d \tau \leq$ $N^{2}$

To prove the estimate (??), we observe that the equations $(2.2)_{i}$ and $(2.2)_{i i}$, imply

$$
\begin{aligned}
& \left\|u_{t}^{n+1}\right\|^{2} \leq C\left(\left\|A u^{n+1}\right\|^{2}+\|f\|^{2}+\left\|u^{n} \cdot \nabla u^{n+1}\right\|^{2}+\left\|h^{n} \cdot \nabla h^{n+1}\right\|^{2}\right), \\
& \left\|h_{t}^{n+1}\right\|^{2} \leq C\left(\left\|A h^{n+1}\right\|^{2}+\left\|u^{n} \cdot \nabla h^{n+1}\right\|^{2}+\left\|h^{n} \cdot \nabla u^{n+1}\right\|^{2}\right)
\end{aligned}
$$

By using the previous relations, we have

$$
\begin{aligned}
\int_{0}^{t}\left\|u_{t}^{n+1}(s)\right\|^{2} d s \leq & C \int_{0}^{t}\left(\left\|A u^{n+1}(s)\right\|^{2}+\|f(s)\|^{2}\right. \\
& \left.+\left\|u^{n} \cdot \nabla u^{n+1}\right\|^{2}+\left\|h^{n} \cdot \nabla h^{n+1}\right\|^{2}\right) d s \\
\int_{0}^{t}\left\|h_{t}^{n+1}(s)\right\|^{2} d s \leq & C \int_{0}^{t}\left(\left\|A h^{n+1}(s)\right\|^{2}+\left\|u^{n} \cdot \nabla h^{n+1}\right\|^{2}\right. \\
& \left.+\left\|h^{n} \cdot \nabla u^{n+1}\right\|^{2}\right) d s .
\end{aligned}
$$


The estimate (3.3) and the Sobolev embedding $H^{2} \hookrightarrow L^{\infty}$ imply

$$
\left\|u^{n} \cdot \nabla u^{n+1}\right\|^{2} \leq\left\|u^{n}\right\|_{L^{\infty}}^{2}\left\|\nabla u^{n+1}\right\|^{2} \leq C\left\|A u^{n}\right\|^{2} .
$$

In the same way, we find

$$
\begin{aligned}
\left\|h^{n} \cdot \nabla h^{n+1}\right\|^{2} & \leq C\left\|A h^{n}\right\|^{2}, \\
\left\|u^{n} \cdot \nabla h^{n+1}\right\|^{2} & \leq C\left\|A u^{n}\right\|^{2}, \\
\left\|h^{n} \cdot \nabla u^{n+1}\right\|^{2} & \leq C\left\|A h^{n}\right\|^{2} .
\end{aligned}
$$

Consequently, the inequalities (3.14), become

$$
\begin{aligned}
\int_{0}^{t}\left\|u_{t}^{n+1}(s)\right\|^{2} d s & \leq C \int_{0}^{t}\left(\left\|A u^{n+1}(s)\right\|^{2}+\|f(s)\|^{2}\right. \\
& +\left\|A u^{n}(s)\right\|^{2}+\left\|A h^{n}(s)\right\|^{2} d s \\
& \leq C \frac{\alpha}{\nu} M_{1}+C\|f\|_{L^{2}(Q)}^{2}
\end{aligned}
$$

and

$$
\begin{aligned}
\int_{0}^{t}\left\|h_{t}^{n+1}(s)\right\|^{2} d s & \leq C \int_{0}^{t}\left(\left\|A h^{n+1}(s)\right\|^{2}+\left\|A u^{n}(s)\right\|^{2}\right. \\
& +\left(\left\|A h^{n}(s)\right\|^{2}\right) d s \\
& \leq C .
\end{aligned}
$$

This completes the proof of the Lemma.

\section{Remark 3.4 :}

The Lemmas 3.1, 3.2 and 3.3 are true again if $u(0) \neq 0, h(0) \neq 0$ with $u_{0} \in V$ and $h_{0} \in V$.

\section{Remark 3.5 :}

By using arguments of compactness is it possible to show that the estimates given in the Lemmas $3.1,3.2$ and 3.3 are sufficient to show that the approximate solution $\left(u^{n}, h^{n}\right)$ converges to a unique strong solution of the problem (1.1)-(1.2). Let us observe that by using these arguments Boldrini and Rojas-Medar [2] do not obtain the convergence rates. In the next section we will prove the convergence of approximate solution by other arguments. 


\section{Lemma 3.6 :}

If $f, f_{t} \in L^{2}(Q)$, then the approximate solutions $\left(u^{n}, h^{n}\right)$ satisfy, uniformly in $n$, the following estimates

$$
\begin{aligned}
& \sup _{t}\left\{\left\|u_{t}^{n}\right\|+\left\|h_{t}^{n}\right\|\right\} \leq M_{2}, \\
& \int_{0}^{t}\left(\left\|\nabla u_{t}^{n}\right\|^{2}+\left\|\nabla h_{t}^{n}\right\|^{2}\right) d s \leq M_{3}, \\
& \sup _{t}\left\{\left\|A u^{n}\right\|+\left\|A h^{n}\right\|\right\} \leq M_{4}, \\
& \int_{0}^{t}\left(\left\|u_{t t}^{n}\right\|_{V^{*}}^{2}+\left\|h_{t t}^{n}\right\|_{V^{*}}^{2}\right) d s \leq M_{5}
\end{aligned}
$$

where $M_{i}, i=2, \ldots, 5$ are positive constants independent of $n$, and depending on $\partial \Omega, \nu, \gamma, \alpha,\|f\|_{L^{2}(Q)},\left\|f_{t}\right\|_{L^{2}(Q)}$ and $M_{1}$ as in the Lemma 3.2 .

\section{Proof. :}

We differentiate $(2.2)_{i}$ and $(2.2)_{i i}$ with respect to $t$ and we multiply by $u_{t}^{n+1}$ and $h_{t}^{n+1}$ and integrating over $\Omega$. We obtain

$$
\begin{aligned}
& \frac{\alpha}{2} \frac{d}{d t}\left\|u_{t}^{n+1}\right\|^{2}+\nu\left\|\nabla u_{t}^{n+1}\right\|^{2} \\
& \quad=\left(\alpha f_{t}, u_{t}^{n+1}\right)-\left(u_{t}^{n} \cdot \nabla u^{n+1}, u_{t}^{n+1}\right)+\left(h_{t}^{n} \cdot \nabla h^{n+1}, u_{t}^{n+1}\right)+\left(h^{n} \cdot \nabla h_{t}^{n+1}, u_{t}^{n+1}\right) \\
& \quad(3.19) \\
& \frac{1}{2} \frac{d}{d t}\left\|h_{t}^{n+1}\right\|^{2}+\gamma\left\|\nabla h_{t}^{n+1}\right\|^{2} \\
& \quad=\left(h_{t}^{n} \cdot \nabla u^{n+1}, h_{t}^{n+1}\right)+\left(h^{n} \cdot \nabla u_{t}^{n+1}, h_{t}^{n+1}\right)-\left(u_{t}^{n} \cdot \nabla h^{n+1}, h_{t}^{n+1}\right)
\end{aligned}
$$

We now estimate the right-hand sides of the above equalities as follows,

$$
\begin{aligned}
\left|\left(\alpha f_{t}, u_{t}^{n+1}\right)\right| & \leq \frac{\alpha^{2}}{2}\left\|f_{t}\right\|^{2}+\frac{1}{2}\left\|u_{t}^{n+1}\right\|^{2} \\
\left|\left(u_{t}^{n} \cdot \nabla u^{n+1}, u_{t}^{n+1}\right)\right| & \leq\left\|u_{t}^{n}\right\|\left\|\nabla u^{n+1}\right\|_{L^{4}}\left\|u_{t}^{n+1}\right\|_{L^{4}} \\
& \leq C_{\varepsilon}\left\|A u^{n+1}\right\|^{2}\left\|u_{t}^{n}\right\|^{2}+\varepsilon\left\|\nabla u_{t}^{n+1}\right\|^{2}, \\
\left|\left(h_{t}^{n} \cdot \nabla h^{n+1}, u_{t}^{n+1}\right)\right| & \leq\left\|h_{t}^{n}\right\|\left\|\nabla h^{n+1}\right\| L_{L^{4}}\left\|u_{t}^{n+1}\right\|_{L^{4}} \\
& \leq C_{\varepsilon}\left\|A h^{n+1}\right\|^{2}\left\|h_{t}^{n}\right\|^{2}+\varepsilon\left\|\nabla u_{t}^{n+1}\right\|, \\
\left|\left(h_{t}^{n} \cdot \nabla u^{n+1}, h_{t}^{n+1}\right)\right| & \leq\left\|h_{t}^{n}\right\|\left\|\nabla u^{n+1}\right\| L_{L^{4}}\left\|h_{t}^{n+1}\right\| L^{4} \\
& \leq C_{\delta}\left\|A u^{n+1}\right\|^{2}\left\|h_{t}^{n}\right\|^{2}+\delta\left\|\nabla h_{t}^{n+1}\right\|^{2},
\end{aligned}
$$




$$
\begin{aligned}
\left|\left(u_{t}^{n} \cdot \nabla h^{n+1}, h_{t}^{n+1}\right)\right| & \leq\left\|u_{t}^{n}\right\|\left\|\nabla h^{n+1}\right\| L_{L^{4}}\left\|h_{t}^{n+1}\right\|_{L^{4}} \\
& \leq C_{\delta}\left\|A h^{n+1}\right\|^{2}\left\|u_{t}^{n}\right\|^{2}+\delta\left\|\nabla h_{t}^{n+1}\right\|^{2} \\
\left(h^{n} \cdot \nabla h_{t}^{n+1}, u_{t}^{n+1}\right) & +\left(h^{n} \cdot \nabla u_{t}^{n+1}, h_{t}^{n+1}\right)=0 .
\end{aligned}
$$

By adding the equalities $(3.19)_{i}$ and $(3.19)_{i i}$ and using the above estimate with $\varepsilon=\frac{v}{4}, \delta=\frac{u}{4}$, we get

$$
\begin{aligned}
& \frac{1}{2} \frac{d}{d t}\left(\alpha\left\|u_{t}^{n+1}\right\|\left\|^{2}+\right\| h_{t}^{n+1} \|^{2}\right) \\
& +\frac{\nu}{2}\left\|\nabla u_{t}^{n+1}\right\|^{2}+\frac{\gamma}{2}\left\|\nabla h_{t}^{n+1}\right\|^{2} \\
\leq & \frac{\alpha^{2}}{2}\left\|f_{t}\right\|^{2}+\frac{1}{2}\left\|u_{t}^{n+1}\right\|^{2} \\
& +C\left(\left\|A u^{n+1}\right\|^{2}+\left\|A h^{n+1}\right\|^{2}\right)\left(\left\|u_{t}^{n}\right\|^{2}+\left\|h_{t}^{n}\right\|^{2}\right) .
\end{aligned}
$$

By integrating the last inequality from 0 to $t$, we obtain

$$
\alpha\left\|u_{t}^{n+1}(t)\right\|^{2}+\left\|h_{t}^{n+1}(t)\right\|^{2}+\nu \int_{0}^{t}\left\|\nabla u_{t}^{n+1}(s)\right\|^{2} d s+\gamma \int_{0}^{t}\left\|\nabla h_{t}^{n+1}(s)\right\|^{2} d s
$$

$$
\begin{aligned}
\leq & C\left\|f_{t}\right\|_{L^{2}(Q)}^{2}+C \int_{0}^{t}\left\|u_{t}^{n+1}(s)\right\|^{2} d s \\
& +C \int_{0}^{t}\left(\left\|A u^{n+1}(s)\right\|^{2}+\left\|A h^{n+1}(s)\right\|^{2}\right)\left(\left\|u_{t}^{n}(s)\right\|^{2}+\left\|h_{t}^{n}(s)\right\|^{2}\right) d s \\
\leq & \tilde{M}_{1}+C \int_{0}^{t}\left(\left\|A u^{n+1}(s)\right\|^{2}+\left\|A h^{n+1}(s)\right\|^{2}\right)\left(\left\|u_{t}^{n}(s)\right\|^{2}+\left.\left\|h_{t}^{n}(s)\right\|\right|^{2}\right) d s,
\end{aligned}
$$

where $\tilde{M}_{1}=C\left[\|f\|_{L^{2}(Q)}^{2}+\left\|f_{t}\right\|_{L^{2}(Q)}^{2}+\frac{\alpha}{\nu} M_{1}\right]$.

The equalities $(2.2)_{i}$ and $(2.2)_{i i}$ imply

$$
\gamma\left\|A h^{n+1}\right\| \leq\left\|h_{t}^{n+1}\right\|+\left\|u^{n} \cdot \nabla h^{n+1}\right\|+\left\|h^{n} \cdot \nabla u^{n+1}\right\| .
$$

We recall the following inequality

$$
\|\phi\|_{L^{3}} \leq C\|\phi\|^{1 / 2}\|\nabla \phi\|^{1 / 2}
$$

By applying this inequality, we have 


$$
\begin{aligned}
\left\|u^{n} \cdot \nabla u^{n+1}\right\| & \leq\left\|u^{n}\right\|_{L^{6}}\left\|\nabla u^{n+1}\right\|_{L^{3}} \\
& \leq C\left\|\nabla u^{n}\right\|\left\|\nabla u^{n+1}\right\|^{1 / 2}\left\|A u^{n+1}\right\|^{1 / 2} \\
& \leq C\left\|\nabla u^{n}\right\|^{2}\left\|\nabla u^{n+1}\right\|+\frac{\nu}{4}\left\|A u^{n+1}\right\|,
\end{aligned}
$$

In the same way, we obtain

$$
\begin{aligned}
& \left\|h^{n} \cdot \nabla h^{n+1}\right\| \leq C\left\|\nabla h^{n}\right\|^{2}\left\|\nabla h^{n+1}\right\|+\frac{\gamma}{4}\left\|A h^{n+1}\right\| \\
& \left\|u^{n} \cdot \nabla h^{n+1}\right\| \leq C\left\|\nabla u^{n}\right\|^{2}\left\|\nabla h^{n+1}\right\|+\frac{\gamma}{4}\left\|A h^{n+1}\right\| \\
& \left\|h^{n} \cdot \nabla u^{n+1}\right\| \leq C\left\|\nabla h^{n}\right\|^{2}\left\|\nabla u^{n+1}\right\|+\frac{\nu}{4}\left\|A u^{n+1}\right\|
\end{aligned}
$$

By adding the inequalities (??) and (??), and using the estimates gives in the Lemma 3.2 together with the last estimates, we obtain

$$
\nu\left\|A u^{n+1}\right\|^{2}+\gamma\left\|A h^{n+1}\right\|^{2} \leq C_{1}+C\left(\alpha\left\|u_{t}^{n+1}\right\|^{2}+\left\|h_{t}^{n+1}\right\|^{2}\right)
$$

Consequently, by using this last estimate in the inequality (3.20), we obtain

$$
\begin{aligned}
& \alpha\left\|u_{t}^{n+1}(t)\right\|^{2}+\left\|h_{t}^{n+1}(t)\right\|^{2}+\nu \int_{0}^{t}\left\|\nabla u_{t}^{n+1}(s)\right\|^{2} d s+\gamma \int_{0}^{t}\left\|\nabla h_{t}^{n+1}(s)\right\|^{2} d s \\
& \leq \tilde{M}_{1}+C \int_{0}^{t}\left(\left\|u_{t}^{n}(s)\right\|^{2}+\left\|h_{t}^{n}(s)\right\|^{2}\right) d s \\
& \quad+C \int_{0}^{t}\left(\alpha\left\|u_{t}^{n+1}(s)\right\|^{2}+\left\|h_{t}^{n+1}(s)\right\|^{2}\right)\left(\left\|u_{t}^{n}(s)\right\|^{2}+\left\|h_{t}^{n}(s)\right\|^{2}\right) d s \\
& \leq M+C \int_{0}^{t}\left(\alpha\left\|u_{t}^{n+1}(s)\right\|^{2}+\left\|h_{t}^{n+1}(s)\right\|^{2}\right)\left(\left\|u_{t}^{n}(s)\right\|^{2}+\left\|l_{t}^{n}(s)\right\|^{2}\right) d s
\end{aligned}
$$

with $M=C \tilde{M}_{1}+\frac{C \alpha}{\gamma} M_{1}$, where we used the estimate (3.5) given in the Lemma 3.2.

By applying the Gronwall inequality, we obtain the estimates (3.15) and (3.16) given in the Lemma.

The estimate (3.17) follow from inequalities (3.23) and (3.15).

To prove (3.18), we observe that by differentiating $(2.2)_{i}$ and $(2.2)_{i i}$ with respect to $t$, we obtain

$$
\begin{aligned}
\alpha u_{t t}^{n+1} & =P\left(\alpha f_{t}\right)-P\left(h^{n} \cdot \nabla h^{n+1}\right)_{t}-P\left(u^{n} \cdot \nabla u^{n+1}\right)_{t}-\nu A u_{t}^{n+1} \\
h_{t t}^{n+1} & =P\left(h^{n} \cdot \nabla u^{n+1}\right)_{t}-P\left(u^{n} \cdot \nabla h^{n+1}\right)_{t}-\gamma A h_{t}^{n+1} .
\end{aligned}
$$


Consequently,

$$
\begin{aligned}
\alpha\left\|u_{i t}^{n+1}\right\|_{V^{*}}^{2} d s & \leq \alpha \int_{0}^{t}\left\|f_{t}\right\|^{2} d s+\int_{0}^{t}\left\|\left(h^{n} \cdot \nabla h^{n+1}\right)_{t}\right\|_{V^{*}} d s \\
& +\int_{0}^{t}\left\|\left(u^{n} \cdot \nabla u^{n+1}\right)_{t}\right\|_{V^{*}}^{2} d s+\nu \int_{0}^{t} \| A u_{t}^{n+1} d s
\end{aligned}
$$

The same result follows for $\int_{0}^{l}\left\|h_{t t}^{n+1}\right\|_{V^{*}}^{2} d s$.

We also note that

$$
\begin{aligned}
\left\|A u_{t}^{n+1}\right\|_{V^{*}} & =\sup _{\|v\|_{\mathbf{V}} \leq 1}\left|\left\langle A u_{t}^{n+1}, v\right\rangle\right| \\
& =\sup _{\|v\|_{\mathbf{V}} \leq 1}\left|\left\langle\nabla u_{t}^{n+1}, \nabla v\right\rangle\right| \\
& \leq\left\|\nabla u_{t}^{n+1}\right\|,
\end{aligned}
$$

so, by using the estimate (3.16), we get

$$
\int_{0}^{t}\left\|A u_{t}^{n+1}\right\|_{V^{*}}^{2} d s \leq C \int_{0}^{t}\left\|\nabla u_{t}^{n+1}\right\|^{2} d s \leq C .
$$

The other terms in (3.25) are estimates in the same way. This completes the proof of the Lemma.

\section{Error Estimates}

In this section, we prove several convergence rate bounds for the approximate solutions. The following Lenma will be fundamental in our future arguments.

\section{Lemma 4.1 :}

Let be $0 \leq \alpha_{1}(t) \leq M$ for all $t \in[0, T]$ and let us assume that the following inequality is true for all $p \geq 2$

$$
\alpha_{p}(t) \leq C \int_{0}^{t} \alpha_{p-1}(s) d s
$$

Then,

$$
\alpha_{p}(t) \leq M \frac{(C t)^{p-1}}{(p-1) !} \leq M \frac{(C T)^{p-1}}{(p-1) !}
$$

for all $t \in[0, T]$ and $p \geq 2$. 


\section{Proof. :}

In fact, we have

$$
\begin{aligned}
\alpha_{p}(t) & \leq C \int_{0}^{t} C \int_{0}^{t_{1}} \alpha_{p-2}\left(t_{2}\right) d t_{2} d t_{1} \\
& \leq C^{2} \int_{0}^{t} \int_{0}^{t_{1}} C \int_{0}^{t_{2}} \alpha_{p-3}\left(t_{3}\right) d t_{3} d t_{2} d t_{1} \\
& \vdots \\
& \leq C^{p-1} \int_{0}^{t} \int_{0}^{t_{1}} \cdots \int_{0}^{t_{p-2}} \alpha_{1}\left(t_{p-1}\right) d t_{p-1} \cdots d t_{1} \\
& \leq M C^{p-1} \int_{0}^{t} \int_{0}^{t_{1}} \cdots \int_{0}^{t_{p-2}} d t_{p-1} \cdots d t_{1} \\
& \equiv M \frac{(C t)^{p-1}}{(p-1) !}
\end{aligned}
$$

Let be

$$
\begin{aligned}
& u^{n, k}(t)=u^{k+n}(t)-u^{n}(t) \\
& h^{n, k}(t)=h^{n+k}(t)-h^{n}(t) .
\end{aligned}
$$

Then, the following equations are satisfied by $u^{n, k}$ and $h^{n, k}$

$$
\begin{aligned}
\alpha u_{t}^{n, k}+\nu A u^{n, k}= & P\left(h^{n-1+k} \cdot \nabla h^{n, k}+h^{n-1, k} \cdot \nabla h^{n}\right. \\
& \left.-u^{n-1+k} \cdot \nabla u^{n, k}-u^{n-1, k} \cdot \nabla u^{n}\right) \\
h_{t}^{n, k}+\gamma A h^{n, k}= & P\left(h^{n-1+k} \cdot \nabla u^{n, k}+h^{n-1, k} \cdot \nabla u^{n}\right. \\
& \left.-u^{n-1+k} \cdot \nabla h^{n, k}-u^{n-1, k} \cdot \nabla h^{n}\right)
\end{aligned}
$$

or equivalently,

$$
\begin{array}{r}
\left(u_{t}^{n, k}, v\right)+\nu\left(\nabla u^{n, k}, \nabla v\right)=\left(h^{n-1+k} \cdot \nabla h^{n, k}, v\right)+\left(h^{n-1, k} \cdot \nabla u^{n}, v\right) \\
-\left(u^{n-1+k} \cdot \nabla u^{n, k}, v\right)-\left(u^{n-1, k} \cdot \nabla u^{n}, \varphi v\right)
\end{array}
$$

$$
\begin{aligned}
\left(h_{t}^{n, k}, \varphi\right)+\gamma\left(\nabla h^{n, k}, \nabla \varphi\right) & =\left(h^{n-1+k} \cdot \nabla u^{n, k}, \varphi\right)+\left(h^{n-1, k} \cdot \nabla u^{n}, \varphi\right) \\
& -\left(u^{n-1+k} \cdot \nabla h^{n, k}, \varphi\right)-\left(u^{n-1, k} \cdot \nabla h^{n}, \varphi\right)
\end{aligned}
$$

for all $v, \varphi \in V$. 
With these notations, we state:

\section{Lemma 4.2 :}

$$
\begin{aligned}
& \alpha\left\|u^{n, k}(t)\right\|^{2}+\left\|h^{n, k}(t)\right\|^{2}+\nu \int_{0}^{t}\left\|\nabla u^{n, k}(s)\right\|^{2} d s+\gamma \int_{0}^{l}\left\|\nabla h^{n, k}(s)\right\|^{2} d s \\
& \leq C \int_{0}^{l}\left(\left\|A u^{n}(s)\right\|+\left\|A h^{n}(s)\right\|\right)\left(\alpha\left\|u^{n-1, k}(s)\right\|^{2}+\left\|h^{n-1, k}(s)\right\|^{2}\right) d s
\end{aligned}
$$

\section{Proof. :}

Setting $v=u^{n, k}$ and $\varphi=h^{n, k}$ in (4.6) and (4.7), we get

$$
\begin{aligned}
\frac{\alpha}{2} \frac{d}{d t}\left\|u^{n, k}\right\|^{2}+\nu\left\|\nabla u^{n, k}\right\|^{2} & =\left(h^{n-1+k} \cdot \nabla h^{n, k}, u^{n, k}\right) \\
& +\left(h^{n-1, k} \cdot \nabla h^{n}, u^{n, k}\right)-\left(u^{n-1, k} \cdot \nabla u^{n}, u^{n, k}\right) \\
\frac{1}{2} \frac{d}{d t}\left\|h^{n, k}\right\|^{2}+\gamma\left\|\nabla h^{n, k}\right\|^{2} & =\left(h^{n-1+k} \cdot \nabla u^{n, k}, h^{n, k}\right) \\
+ & \left(h^{n-1, k} \cdot \nabla u^{n}, h^{n, k}\right)-\left(u^{n-1, k} \cdot \nabla h^{n}, h^{n, k}\right) .
\end{aligned}
$$

Adding the above equalities and observing that

$$
\left(h^{n-1+k} \cdot \nabla h^{n, k}, u^{n, k}\right)+\left(h^{n-1+k} \cdot \nabla u^{n, k}, h^{n, k}\right)=0,
$$

we get

$$
\begin{aligned}
& \frac{1}{2} \frac{d}{d t}\left(\alpha\left\|u^{n, k}\right\|^{2}+\left\|h^{n, k}\right\|^{2}\right)+\gamma\left\|\nabla u^{n, k}\right\|^{2}+\gamma\left\|\nabla h^{n, k}\right\|^{2}= \\
& \left(h^{n-1, k} \nabla h^{n}, u^{n, k}\right)-\left(u^{n-1, k} \nabla u^{n}, u^{n, k}\right) \\
& +\left(h^{n-1, k} \nabla u^{n}, h^{n, k}\right)-\left(u^{n-1, k} \nabla h^{n}, h^{n, k}\right)
\end{aligned}
$$

We now estimate the right-hand side of the above equality as follows:

Using the Hölder inequality and estimates (3.3) and (3.17), we have

$$
\begin{aligned}
\left|\left(h^{n-1, k} \nabla h^{n}, u^{n, k}\right)\right| & \leq\left\|h^{n-1, k}\right\|\left\|\nabla h^{n}\right\|_{L^{3}}\left\|u^{n, k}\right\|_{L^{6}} \\
& \leq C_{3}\left\|h^{n-1, k}\right\|^{2}\left\|A h^{n}\right\|\left\|\nabla h^{n}\right\|+\varepsilon\left\|\nabla u^{n, k}\right\|^{2} \\
& \leq C_{3}\left\|A h^{n}\right\|\left\|h^{n-1, k}\right\|^{2}+\varepsilon\left\|\nabla u^{n, k}\right\|^{2} .
\end{aligned}
$$

Analogously,

$$
\begin{aligned}
& \left|\left(u^{n-1, k} \nabla u^{n}, u^{n, k}\right)\right| \leq C_{\varepsilon}\left\|A u^{n}\right\|\left\|u^{n-1, k}\right\|^{2}+\varepsilon\left\|\nabla u^{n, k}\right\|^{2}, \\
& \left|\left(h^{n-1, k} \nabla u^{n}, h^{n, k}\right)\right| \leq C_{\delta}\left\|A u^{n}\right\|\left\|h^{n-1, k}\right\|^{2}+\delta \| \nabla h^{n, k}||^{2}, \\
& \left|\left(u^{n-1, k} \nabla h^{n}, h^{n, k}\right)\right| \leq C_{\delta}\left\|A h^{n}\right\|\left\|u^{n-1, k}\right\|^{2}+\delta\left\|\nabla h^{n, k}\right\|^{2} .
\end{aligned}
$$


Taking $\varepsilon=\frac{\nu}{4}$ and $\delta=\frac{\gamma}{4}$ in the above estinates, we obtain in (4.8)

$$
\begin{aligned}
& \frac{d}{d t}\left(\alpha\left\|u^{n, k}\right\|^{2}+\left\|h^{n, k}\right\|^{2}\right)+\nu\left\|\nabla u^{n, k}\right\|^{2}+\gamma\left\|\nabla h^{n, k}\right\|^{2} \\
& \quad \leq C\left(\left\|A u^{n}\right\|+\left\|A h^{n}\right\|\right)\left(\alpha\left\|u^{n-1, k}\right\|^{2}+\left\|h^{n-1, k}\right\|^{2}\right) .
\end{aligned}
$$

By integrating from 0 to $t$, we obtain the desired result.

\section{Lemma 4.3 :}

$$
\begin{aligned}
& \alpha\left\|\nabla u^{n, k}(t)\right\|^{2}+\left\|\nabla h^{n, k}(t)\right\|^{2}+\nu \int_{0}^{t}\left\|A u^{n, k}(s)\right\|^{2} d s+\gamma \int_{0}^{t}\left\|A h^{n, k}(s)\right\|^{2} d s \\
& \leq C \int_{0}^{t}\left(\left\|A u^{n}\right\|+\left\|A h^{n}\right\|\right)\left(\alpha\left\|\nabla u^{n-1, k}\right\|^{2}+\left\|\nabla h^{n-1, k}\right\|^{2}\right) d s .
\end{aligned}
$$

\section{Proof. :}

Setting $v=A u^{n, k}$ in (4.6) and $\varphi=A h^{n, k}$ in (4.7), we obtain

$$
\begin{gathered}
\alpha 2 \frac{d}{d t}\left\|\nabla u^{n, k}\right\|^{2}+\nu\left\|A u^{n, k}\right\|^{2}=\left(h^{n-1+k} \cdot \nabla h^{n, k}, A u^{n, k}\right)+\left(h^{n-1, k} \cdot \nabla h^{n}, A u^{n, k}\right) \\
-\left(u^{n-1+k} \cdot \nabla u^{n, k}, A u^{n, k}\right)-\left(u^{n-1, k} \cdot \nabla u^{n}, A u^{n, k}\right), \\
\frac{1}{2} \frac{d}{d t}\left\|\nabla h^{n, k}\right\|^{2}+\gamma\left\|A h^{n, k}\right\|^{2}=\frac{1}{2} \frac{d}{d t}\left\|\nabla h^{n, k}\right\|^{2}+\gamma\left\|A h^{n, k}\right\|^{2} \\
-\left(u^{n-1+k} \cdot \nabla h^{n, k}, A h^{n, k}\right)-\left(u^{n-1, k} \cdot \nabla h^{n}, A h^{n, k}\right) .
\end{gathered}
$$

We now estimate the right-hand sides of the above equalities as follows: by using the Hölder inequality together with the Sobolev's embedding $H^{2} \hookrightarrow L^{\infty}$ and $H^{1} \hookrightarrow L^{p} 1 \leq p \leq 6$, we have

$$
\begin{aligned}
\left|\left(h^{n-1+k} \cdot \nabla h^{n, k}, A u^{n, k}\right)\right| & \leq\left\|h^{n-1+k}\right\|_{L^{\infty}}\left\|\nabla h^{n, k}\right\|\left\|A u^{n, k}\right\| \\
& \leq C_{\varepsilon}\left\|A h^{n-1+k}\right\|^{2}\left\|\nabla h^{n, k}\right\|^{2}+\varepsilon\left\|A u^{n, k}\right\|^{2}, \\
\left|\left(h^{n-1, k} \cdot \nabla u^{n}, A h^{n, k}\right)\right| & \leq\left\|h^{n-1, k} \cdot \nabla u^{n}\right\|\left\|A h^{n, k}\right\| \\
& \leq C_{\delta}\left\|h^{n-1, k}\right\|_{L^{6}}^{2}\left\|\nabla u^{n}\right\|_{L^{3}}^{2}+\delta\left\|A h^{n, k}\right\|^{2} \\
& \leq C_{\delta}\left\|\nabla h^{n-1, k}\right\|^{2}\left\|A u^{n}\right\|+\delta\left\|A h^{n, k}\right\|^{2} .
\end{aligned}
$$


The other terms in the above equalities can be estimate in a similar way; consequently, we obtain the following differential inequality

$$
\begin{aligned}
& \frac{d}{d t}\left(\alpha\left\|\nabla u^{n, k}\right\|^{2}+\left\|\nabla h^{n, k}\right\|^{2}\right)+\nu\left\|A u^{n, k}\right\|^{2}+\gamma\left\|A h^{n, k}\right\|^{2} \\
& \leq C\left(\left\|A u^{n-1+k}\right\|^{2}+\left\|A h^{n-1+k}\right\|^{2}\right)\left(\alpha\left\|\nabla u^{n, k}\right\|^{2}+\left\|\nabla h^{n, k}\right\|^{2}\right) \\
&+C\left(\left\|A u^{n}\right\|+\left\|A h^{n}\right\|\right)\left(\alpha\left\|\nabla u^{n-1, k}\right\|^{2}+\left\|\nabla h^{n-1, k}\right\|^{2}\right)
\end{aligned}
$$

We integrate from 0 to $t$, and we use the Gronwall's inequality to get

$$
\begin{aligned}
& \alpha\left\|\nabla u^{n, k}(t)\right\|^{2}+\left\|\nabla h^{n, k}(t)\right\|^{2}+\nu \int_{0}^{t}\left\|A u^{n, k}(s)\right\|^{2} d s+\gamma \int_{0}^{t}\left\|A h^{n, k}(s)\right\|^{2} d s \\
& \leq C \int_{0}^{t}\left(\left\|A u^{n}\right\|+\left\|A h^{n}\right\|\right)\left(\alpha\left\|\nabla u^{n-1, k}\right\|^{2}+\left\|\nabla h^{n-1, k}\right\|^{2}\right) d s \times \\
& \times \exp C \int_{0}^{t}\left(\left\|A u^{n-1+k}\right\|^{2}+\left\|A h^{n-1+k}\right\|^{2}\right) d s .
\end{aligned}
$$

By the estimate, (3.4) given in the Lemma 3.2, we have

$$
\left.\int_{0}^{t}\left\|A u^{n-1+k}\right\|^{2}+\left\|A h^{n-1+k}\right\|^{2}\right) d s \leq C .
$$

Hence, we complete the proof of the Lemma.

\section{Corollary 4.4 :}

$$
\begin{aligned}
& \int_{0}^{t}\left(\left\|u_{t}^{n, k}(s)\right\|^{2}+\left\|h_{t}^{n, k}(s)\right\|^{2}\right) d s \\
& \leq C \int_{0}^{t}\left(\left\|A u^{n}(s)\right\|+\left\|A h^{n}(s)\right\|\right)\left(\alpha\left\|\nabla u^{n-1, k}(s)\right\|^{2}+\left\|\nabla h^{n-1, k}(s)\right\|^{2}\right) d s .
\end{aligned}
$$

\section{Theorem 4.5:}

If $f \in L^{2}\left(0, T ; L^{2}(\Omega)\right), u_{0}, h_{0} \in V$ and the hypotheses of the Lemma 3.2 are verified, the approximate solutions $\left(u^{n}, h^{n}\right)$ convergence in the space $L^{2}\left(0, T ; H^{2} \cap V\right)$. The limiting element $(u, h)$ of the given sequence is a solution of problem (2.1) and the solution is unique. The rate of convergence satisfies the inequalities

$$
\sup _{0 \leq t \leq T}\left\{\left\|u^{n}(t)-u(t)\right\|^{2}+\left\|h^{n}(t)-h(t)\right\|^{2}\right\} \leq\left[\frac{\left(M_{6} T\right)^{n-1}}{(n-1) !}\right]^{1 / 2}
$$




$$
\begin{gathered}
\int_{0}^{t}\left(\left\|\nabla u^{n}(s)-\nabla u(s)\right\|^{2}+\left\|\nabla h^{n}(s)-\nabla h(s)\right\|^{2}\right) d s \leq \\
{\left[\frac{\left(M_{7} T\right)^{n-1}}{(n-1) !}\right]^{1 / 2},} \\
\sup _{0 \leq t \leq T}\left\{\left\|\nabla u^{n}(t)-\nabla u(t)\right\|^{2}+\left\|\nabla h^{n}(t)-\nabla h(t)\right\|^{2}\right) \leq \\
{\left[\frac{\left(M_{8} T\right)^{n-1}}{(n-1) !}\right]^{1 / 2},} \\
\int_{0}^{t}\left(\left\|A u^{n}(s)-A u(s)\right\|^{2}+\left\|A h^{n}(s)-A h(s)\right\|^{2}\right) d s \leq \\
{\left[\frac{\left(M_{9} T\right)^{n-1}}{(n-1) !}\right]^{1 / 2},} \\
\int_{0}^{t}\left(\left\|u_{t}^{n}(s)-u_{t}(s)\right\|^{2}+\left\|h_{t}^{n}(s)-h_{t}(s)\right\|^{2}\right) d s \leq \\
{\left[\frac{\left(M_{10} T\right)^{n-1}}{(n-1) !}\right]}
\end{gathered}
$$

where $M_{i}, i=6, \ldots, 10$, are positive constants, independent of $n$, and depending of the positive constants given in the Lemmas of the above sections.

\section{Proof. :}

We set

$$
\phi_{n, k}(t)=\alpha\left\|u^{n, k}(t)\right\|^{2}+\left\|h^{n, k}(t)\right\|^{2},
$$

then the Lemma 4.2 implies

$$
\phi_{n, k}(t) \leq C \int_{0}^{t} \varphi_{n}(s) \phi_{n-1, k}(s) d s
$$

with $\varphi_{n}(t)=\left\|A u^{n}(t)\right\|+\left\|A h^{n}(t)\right\|$.

By using the Cauchy-Schwarz inequality and the estimate (3.4), we obtain

$$
\phi_{n, k}(t) \leq C_{0}\left[\int_{0}^{t} \phi_{n-1, k}^{2}(s) d s\right]^{1 / 2}
$$

with $C_{0}$ positive constant independent of $n$ and $k$ or, equivalently,

$$
\phi_{n, k}^{2}(t) \leq C_{0}^{2} \int_{0}^{t} \phi_{n-1, k}^{2}(s) d s .
$$

By setting $\alpha_{n}(t)=\phi_{n, k}^{2}(t)$ and observing that

$$
0 \leq \alpha_{1}(t) \leq \alpha\left\|u^{1, k}\right\|^{2}+\left\|h^{1, k}\right\|^{2} \leq M
$$


by the estimate (??); by applying the Lemma 4.1, we obtain

$$
\alpha_{n, k}(t) \leq C_{0}^{2(n-1)} \frac{t^{n-1}}{(n-1) !}
$$

consequently,

$$
\phi_{n, k}(t) \leq M C_{0}^{n-1}\left[\frac{t^{n-1}}{(n-1) !}\right]^{1 / 2} .
$$

The series

$$
\sum_{n=1}^{\infty}\left[\frac{\left(C_{0}^{2} T\right)^{n-1}}{(n-1) !}\right]^{1 / 2}
$$

converge, and hence

$$
\lim _{n \rightarrow \infty}\left[\frac{\left(C_{0}^{2} T\right)^{n-1}}{(n-1) !}\right]^{1 / 2}=0 .
$$

The Lemma 4.2 also implies

$$
\begin{aligned}
& \nu \int_{0}^{t}\left\|\nabla u^{n, k}(s)\right\|^{2} d s+\gamma \int_{0}^{t}\left\|\nabla h^{n, k}(s)\right\|^{2} d s \\
\leq & C\left(\int_{0}^{t}\left(\alpha\left\|u^{n-1, k}(s)\right\|^{2}+\left\|h^{n-1, k}(s)\right\|^{2} d s\right)^{1 / 2}\right. \\
\leq & C\left[\int_{0}^{t} \phi_{n-1, k}^{2}(s) d s\right]^{1 / 2} \\
\leq & C\left[\int_{0}^{t} C_{0}^{2(n-2)} \frac{s^{n-2}}{(n-2) !} d s\right]^{1 / 2} \\
\leq & C C_{0}^{(n-2)}\left[\frac{t^{n-1}}{(n-1) !}\right]^{1 / 2} \\
\leq & C\left[\frac{\left(C_{0}^{2} t\right)^{n-1}}{(n-1) !}\right]^{1 / 2}
\end{aligned}
$$

It follows from (4.15)-(4.16) that $\left(u^{n}, h^{n}\right)$ is a Cauchy sequence in $L^{\infty}(0, T ; H) \cap L^{2}(0, T ; V)$. If $(u, h)$ denotes the limiting element of that sequence, it is easily to show from (4.15) and (4.16), that

$u^{n} \rightarrow u$ strongly in $L^{\infty}(0, T ; H) \cap L^{2}(0, T ; V)$ 


$$
h^{n} \rightarrow h
$$

and the convergence-rate bounds (4.9)-(4.10) can be obtained by taking the limit as $k \rightarrow \infty$ in (4.15) and (4.16).

The Lemma 4.3 implies that

$$
\phi_{n, k}(t)=\alpha\left\|\nabla u^{n, k}(t)\right\|^{2}+\left\|\nabla h^{n, k}(t)\right\|^{2}
$$

which satisfies

$$
\phi_{n, k}(t) \leq C \int_{0}^{t} \varphi_{n}(s) \phi_{n-1, k}(s) d s
$$

where $\varphi_{n}(s)=\left\|A u^{n}(s)\right\|+\left\|A h^{n}(s)\right\|$.

This implies

$$
\phi_{n, k}^{2}(t) \leq C_{0}^{2} \int_{0}^{t} \phi_{n-1, k}^{2}(s) d s
$$

By using the same argument, we obtain

$$
\alpha\left\|\nabla u^{n, k}(t)\right\|^{2}+\left\|\nabla h^{n, k}(t)\right\|^{2} \leq M C_{0}^{n-1}\left[\frac{t^{n-1}}{(n-1) !}\right]^{1 / 2}
$$

i.e., the sequence $\left(u^{n}, h^{n}\right)$ is a Cauchy sequence in $L^{\infty}(0, T ; V)$.

The Lemma 4.3 also implies

(4.18) $\gamma \int_{0}^{t}\left\|A u^{n, k}(s)\right\|^{2} d s+\gamma \int_{0}^{t}\left\|A h^{n, k}(s)\right\|^{2} d s \leq \frac{C}{C_{0}}\left[\frac{\left(C_{0}^{2} t\right)^{n-1}}{(n-1) !}\right]^{1 / 2}$

and consequently $\left(u^{n}, h^{n}\right)$ is a Cauchy sequence in $L^{2}(0, T ; D(A))$.

From Corollary 4.4, we have

$$
\int_{0}^{t}\left(\left\|u_{t}^{n, k}(s)\right\|^{2}+\left\|h_{t}^{n, k}(s)\right\|^{2}\right) d s \leq \frac{C}{C_{0}}\left[\frac{\left(C_{0}^{2} t\right)^{n-1}}{(n-1) !}\right]^{1 / 2}
$$

i.e., $u_{t}^{n}$ and $h_{t}^{n}$ are Cauchy sequences in $L^{2}(0, T ; H)$.

With the convergence proved above it is easily to show that $(u, h)$ is a solution of problem.

The convergence-rate bounds (4.11),(4.12) and (4.13) can be obtained by taking the limit as $k \rightarrow \infty$ in inequalities (4.17), (4.18) and (4.19), respectively.

We will show that the solution of problem (1.1)-(1.2) is unique. Let us suppose that there exist another solution $\left(u_{1}, h_{1}\right)$. we put 


$$
\varphi(t)=\alpha\left\|u-u_{1}\right\|^{2}+\left\|h-h_{1}\right\|^{2} .
$$

By the same arguments which yield to (4.14), we obtain the relation

$$
\varphi^{2}(t) \leq C \int_{0}^{t} \varphi^{2}(s) d s
$$

from which it follows, by Gronwall's inequality, that the function $\varphi(t)$ is equal to zero. This proves the theorem.

If, we assume that the given force and initial datum are more regular, we obtain stronger results.

In fact, we have:

\section{Theorem 4.6 :}

If $f, f_{t} \in L^{2}(Q), u_{0}, h_{0} \in V \cap H^{2}(\Omega)$ and the hypotheses of the Lemma 3.2 are true, the following convergence rates are verified

$$
\begin{array}{r}
\left.\sup _{0 \leq t \leq T}\left\{\left\|u^{n}(t)-u(t)\right\|^{2}+\| h^{n}(t)-h(t)\right) \|^{2}\right\} \leq \\
\frac{\left(M M_{11} T\right)^{n-1}}{(n-1) !}, \\
\int_{0}^{t}\left\{\left\|\nabla u^{n}(s)-\nabla u(s)\right\|^{2}+\left\|\nabla h^{n}(s)-\nabla h(s)\right\|^{2}\right\} d s \leq \\
\frac{\left(M I_{12} T\right)^{n-1}}{(n-1) !}, \\
\sup _{0 \leq t \leq T}\left\{\left\|\nabla u^{n}(t)-\nabla u(t)\right\|^{2}+\| \nabla h^{n}(t)-\frac{\left.\nabla h(t) \|^{2}\right\} \leq}{\left(M I_{13} T\right)^{n-1}}\right. \\
\int_{0}^{t}\left\{\left\|A u^{n}(s)-A u(s)\right\|^{2}+\left\|A h^{n}(s)-A h(s)\right\|^{2}\right\} d s \leq \\
\frac{\left(M M_{14} T\right)^{n-1}}{(n-1) !}, \\
\int_{0}^{t}\left\{\left\|u_{t}^{n}(s)-u_{t}(s)\right\|^{2}+\left\|h_{t}^{n}(s)-h_{t}(s)\right\|^{2}\right\} d s \leq \\
\frac{\left(M M_{15} T\right)^{n-1}}{(n-1) !},
\end{array}
$$

where $M_{i}, i=11, \ldots, 15$ are positive constants, independent of $n$, and depending of the positive constants given in the Lemmas of the above sections.

\section{Proof. :}

We set

$$
\phi_{n, k}(t)=\alpha\left\|u^{n, k}(t)\right\|^{2}+\left\|h^{n, k}(t)\right\|^{2}
$$

Then the Lemma 4.2 implies, 


$$
\phi_{n, k}(t) \leq C \int_{0}^{t} \varphi_{n}(s) \phi_{n-1, k}(s) d s
$$

with $\varphi_{n}(t)=\left\|A u^{n}(t)\right\|+\left\|A h^{n}(t)\right\|$.

The Lemma 3.5 implies

$$
\varphi_{n}(t) \leq C \quad \text { for all } t \in[0, T] .
$$

Consequently in (4.25), we have

$$
\phi_{n, k}(t) \leq C \int_{0}^{t} \phi_{n-1, k}(s) d s .
$$

We now use the Lemma 4.1 with $\alpha_{n}(t)=\phi_{n, k}(t)$, to obtain

$$
\alpha\left\|u^{n, k}(t)\right\|^{2}+\left\|h^{n, k}(t)\right\|^{2}=\phi_{n, k}(t) \leq C \frac{(C T)^{n-1}}{(n-1) !} .
$$

By taking the limit $k \rightarrow \infty$, we obtain the estimate (4.20).

Furthermore, the Lemma 4.2 implies

$$
\begin{aligned}
\left\|u^{n+k}-u^{n}\right\|_{L^{2}(0, T ; V)}^{2} \leq \frac{\left(M_{14} T\right)^{n-1}}{(n-1) !} \\
\left\|h^{n+k}-h^{n}\right\|_{L^{2}(0, T ; V)}^{2} \leq \frac{\left(M_{14} T\right)^{n-1}}{(n-1) !} .
\end{aligned}
$$

By taking the limit $k \rightarrow \infty$, we obtain the estimate (4.21).

we now prove the estimate (4.22).

The Lemma 4.3 implies that

$$
\phi_{n, k}(t)=\alpha\left\|\nabla u^{n, k}(t)\right\|^{2}+\left\|\nabla h^{n, k}(t)\right\|^{2}
$$

which satisfies

$$
\phi_{n, k} \leq C \int_{0}^{t} \varphi_{n}(s) \phi_{n-1, k}(s) d s
$$

where $\varphi_{n}(s)=\left\|A u^{n}(s)\right\|+\left\|A h^{n}(s)\right\|$.

By other hand, the Lemma 3.5 implies

$$
\varphi_{n}(t) \leq C \quad \text { for all } t \in[0, T] .
$$

By using the same argument, we obtain 


$$
\alpha\left\|\nabla u^{n, k}(t)\right\|^{2}+\left\|\nabla h^{n, k}(t)\right\|^{2} \leq C \frac{(M t)^{n-1}}{(n-1) !}
$$

The convergence-rate bound is obtained taking the limit $k \rightarrow \infty$.

The estimates (4.23) and (4.24) are easily obtained from the Lemma 4.3 and Corollary 4.4, together with (4.26).

\section{Theorem 4.7 :}

Under the hypotheses of Theorem 4.6, the unique solution obtained in the Theoren 4.5 satisfy $u, h \in C^{1}([0, T] ; H) \cap C([0, T] ; D(A))$ and the following estimates

$$
\begin{aligned}
\left.\sup _{0 \leq t \leq T}\left\{\left\|u_{t}^{n}(t)-u_{t}(t)\right\|^{2}+\| h_{t}^{n}(t)-h_{t}(t)\right) \|^{2}\right\} & \leq \frac{\left(M_{16} T\right)^{n-2}}{(n-2) !} \\
\int_{0}^{t}\left(\left\|\nabla u_{t}^{n}(s)-\nabla u_{t}(s)\right\|^{2}+\left\|\nabla h_{t}^{n}(s)-\nabla h_{t}(s)\right\|^{2}\right\} d s & \leq \frac{\left(M_{17} T\right)^{n-2}}{(n-2) !} \\
\sup _{0 \leq t \leq T}\left\{\left\|A u^{n}(t)-A u(t)\right\|^{2}+\left\|A h^{n}(t)-A h(t)\right\|^{2}\right\} & \leq \frac{\left(M_{18} T\right)^{n-2}}{(n-2) !} \\
\int_{0}^{t}\left\{\left(\left\|u_{t u}^{n}(s)-u_{t t}(s)\right\|_{V^{*}}^{2}+\left\|h_{t t}^{n}-h_{t t}(s)\right\|_{V^{*}}^{2}\right\} d s\right. & \leq \frac{\left(M_{19} T\right)^{n-2}}{(n-2) !},
\end{aligned}
$$

where $M_{i},=i=16, \ldots, 19$, are positive constants, independent of, and depending of the positive constants given in the Lemmas of the above sections.

\section{Proof. :}

We will prove only the two first estimates, the other estimates are similarly obtained.

By differentiating (4.4) and (4.5) with respect to $t$, by taking the product in $L^{2}(\Omega)$ with $u_{t}^{n, k}$ and $h_{t}^{n, k}$, respectively, and adding the results, we obtain 


$$
\begin{aligned}
\frac{1}{2} \frac{d}{d t}\left(\alpha\left\|u_{t}^{n, k}\right\|^{2}\right. & \left.+\left\|h_{t}^{n, k}\right\|^{2}\right)+\gamma \int_{0}^{t}\left\|\nabla u_{t}^{n, k}(s)\right\|^{2} d s+\gamma \int_{0}^{t}\left\|\nabla h_{t}^{n, k}(s)\right\|^{2} d s \\
= & \left(h^{n-1+k} \cdot \nabla h^{n, k}, u_{t}^{n, k}\right)-\left(u_{t}^{n, 1+k} \cdot \nabla u^{n, k}, u_{t}^{n, k}\right) \\
+ & \left(h_{t}^{n-1+k} \cdot \nabla u^{n, k}, h_{t}^{n, k}\right)-\left(u_{t}^{n-1+k} \cdot \nabla h^{n, k}, h_{t}^{n, k}\right) \\
+ & \left(h^{n-1+k} \cdot \nabla h_{t}^{n, k}, u_{t}^{n, k}\right)+\left(h^{n-1+k} \cdot \nabla u_{t}^{n, k}, h_{t}^{n, k}\right) \\
& +\left(h_{t}^{n-1, k} \cdot \nabla h^{n}, u_{t}^{n, k}\right)-\left(u^{n-1, k} \cdot \nabla u^{n}, u_{t}^{n, k}\right) \\
& +\left(h_{t}^{n-1, k} \cdot \nabla u^{n}, h_{t}^{n, k}\right)-\left(u_{t}^{n-1, k} \cdot \nabla h^{n}, h_{t}^{n, k}\right) \\
& +\left(h^{n-1, k} \cdot \nabla h_{t}^{n}, u_{t}^{n, k}\right)-\left(u^{n-1, k} \cdot \nabla u_{t}^{n}, h_{t}^{n, k}\right) \\
& +\left(h^{n-1, k} \cdot \nabla u_{t}^{n}, h_{t}^{n, k}\right)-\left(u^{n-1, k} \cdot \nabla h_{t}^{n}, h_{t}^{n, k}\right) .
\end{aligned}
$$

We now estimate the right-hand side of the above equality.

By using the Hölder and Young inequality, we obtain

$$
\begin{aligned}
\left|\left(h_{t}^{n-1+k} . \nabla h^{n, k}, u_{t}^{n, k}\right)\right| & \leq\left\|\nabla h^{n, k}\right\|\left\|h_{t}^{n-1+k}\right\|_{L^{3}}\left\|u_{t}^{n, k}\right\|_{L^{6}} \\
& \leq\left. C_{\varepsilon}\left\|\nabla h^{n, k}\right\|\right|^{2}\left\|\nabla h_{t}^{n-1+k}\right\|^{2}+\varepsilon\left\|\nabla u_{t}^{n, k}\right\|^{2}, \\
\left|\left(h^{n-1+k} \cdot \nabla h_{t}^{n, k}, u_{t}^{n, k}\right)\right| & \leq\left\|h^{n-1+k}\right\| L_{L^{\infty}}\left\|\nabla h_{t}^{n, k}\right\|\left\|u_{t}^{n, k}\right\| \\
& \leq C_{\delta}\left\|A h^{n-1+k}\right\|^{2}\left\|u_{t}^{n, k}\right\|^{2}+\delta\left\|\nabla h_{t}^{n, k}\right\|^{2}, \\
\left|\left(h_{t}^{n-1, k} . \nabla h^{n}, u_{t}^{n, k}\right)\right| & \leq\left\|h_{t}^{n-1, k}\right\|\left\|\nabla h^{n}\right\|_{L^{3}}\left\|u^{n, k}\right\|_{L^{6}} \\
& \leq C_{\varepsilon}\left\|A h^{n}\right\|^{2}\left\|h_{t}^{n-1, k}\right\|^{2}+\varepsilon\left\|\nabla u^{n, k}\right\|^{2}, \\
\left|\left(h^{n-1, k} \cdot \nabla h_{t}^{n}, u_{t}^{n, k}\right)\right| & \leq\left\|h_{L^{3}}^{n-1, k}\right\|\left\|\nabla h_{t}^{n}\right\|\left\|u_{t}^{n, k}\right\|_{L^{6}} \\
& \leq C_{\varepsilon}\left\|\nabla h^{n-1, k}\right\|^{2}\left\|\nabla h_{t}^{n}\right\|^{2}+\varepsilon\left\|\nabla u^{n, k}\right\|^{2} .
\end{aligned}
$$

The other terms in (4.27) are analogously estimates. Then, by using the above estimates, we obtain the following integral inequality

$$
\begin{aligned}
& \alpha\left\|u_{t}^{n, k}(t)\right\|^{2}+\left\|h_{t}^{n, k}(t)\right\|^{2}+\nu \int_{0}^{t}\left\|\nabla u_{t}^{n, k}(s)\right\|^{2} d s+\gamma \int_{0}^{t}\left\|\nabla h_{t}^{n, k}(s)\right\|^{2} d s \\
\leq & C\left(\left\|\nabla u^{n, k}\right\|^{2}+\left\|\nabla h^{n, k}\right\|^{2}\right)\left(\int_{0}^{t}\left(\left\|\nabla u_{t}^{n-1+k}(s)\right\|^{2}+\left\|\nabla h_{t}^{n-1+k}(s)\right\|^{2}\right) d s\right) \\
& +C\left\|A h^{n-1+k}\right\|^{2} \int_{0}^{t}\left(\left\|u_{t}^{n, k}\right\|^{2}+\left\|h_{t}^{n, k}(s)\right\|^{2}\right) d s \\
& +C\left(\left\|A u^{n}\right\|^{2}+\left\|A h^{n}\right\|^{2}\right) \int_{0}^{t}\left(\left\|u_{t}^{n-1, k}\right\|^{2}+\left\|h_{t}^{n-1, k}\right\|^{2}\right) d s \\
& +C\left(\left\|\nabla u^{n-1, k}\right\|^{2}+\left\|\nabla h^{n-1, k}\right\|^{2}\right) \int_{0}^{t}\left(\left\|\nabla u_{t}^{n}\right\|^{2}+\left\|\nabla h_{t}^{n}\right\|^{2}\right) d s \\
\leq & \frac{(C T)^{n-1}}{(n-1) !}+\frac{(C T)^{n-2}}{(n-2) !}
\end{aligned}
$$




$$
\leq \frac{(C T)^{n-2}}{(n-2) !}
$$

by the estimatos given in Theorem 4.6 and Lemma 3.5 .

\section{Results on the "pressures"}

By using the Amrouche-Girault [1] results on the Stokes problem and the estimates given in the last sections, we obtain the following Propositions.

\section{Proposition 5.1 :}

Under the hypotheses of the Theorem 4.5 , the approximations $\left(p^{n}, w^{n}\right)$ converge in $L^{2}\left(0, T ; H^{1}(\Omega) / \mathbb{R}\right)$. The elcment limit $(p, w) \in L^{2}\left(0, T ; H^{1}(\Omega) / \mathbb{R}\right)$ together with $(u, h)$ obtained in the Theorem 4.5 satisfy the system (1.1)-(1.2). Moreover, the following convergence-rates are true,

$$
\begin{aligned}
\int_{0}^{t}\left\|p^{n}(s)-p(s)\right\|_{H^{1}(\Omega) / \| R}^{2} d s & \leq\left[\frac{\left(M_{20} T\right)^{n-1}}{(n-1) !}\right]^{1 / 2} \\
\int_{0}^{t}\left\|w^{n}(s)-w(s)\right\|_{H^{1}(\Omega) / \Delta k^{2}}^{2} d s & \leq\left[\frac{\left(M_{21} T\right)^{n-1}}{(n-1) !}\right]^{1 / 2}
\end{aligned}
$$

where $p^{n}=\left(p^{*}\right)^{n}+\frac{\mu}{2} h^{n} \cdot h^{n}, p=p^{*}+\frac{\mu}{2} h . h$ and $M_{20}, M_{21}$ are positives constants, independent of $n$, that only depend of the positive constants given in the Lemmas of the above sections.

\section{Proposition 5.2 :}

Under the hypotheses of the Theorem 4.6, $\left(p^{n}, w^{n}\right)$ converge in $L^{\infty}\left(0, T ; H^{1}(\Omega) / \mathbb{R}\right)$ to $(p, w)$.

Moreover, the following convergence rates are true

$$
\begin{aligned}
\int_{0}^{t}\left\|p^{n}(s)-p(s)\right\|_{H^{1}(\Omega) / \mathbb{R}}^{2} d s & \leq \frac{\left(M_{22} T\right)^{n-1}}{(n-1) !} \\
\int_{0}^{t}\left\|w^{n}(s)-w(s)\right\|_{H^{1}(\Omega) / \mathbb{R}}^{2} d s & \leq \frac{\left(M_{23} T\right)^{n-1}}{(n-1) !} \\
\sup _{0 \leq t \leq T}\left\|p^{n}(t)-p(t)\right\|_{H^{1}(\Omega) / \mathbb{R}}^{2} & \leq \frac{\left(M_{24} T\right)^{n-2}}{(n-2) !} \\
\sup _{0 \leq t \leq T}\left\|w^{n}(t)-w(t)\right\|_{H^{1}(\Omega) / \mathbb{R}}^{2} & \leq \frac{\left(M_{25} T\right)^{n-2}}{(n-2) !}
\end{aligned}
$$


where $M_{22}, \ldots, M_{25}$ are positives constants, independent of $n$, and depending on the positive constants given in the Lemmas of the above sections.

Acknowledgments. The authors wish to express their thanks the referce for helpful comments and suggestions which improved the present paper.

\section{References}

[1] Amrouche, G. and Girault, V., On the existence and regularity of the solutions of Stokes problem in arbitrary dimension, Proc. Japan Acad., 67, Ser. A. (1991), 171-175.

[2] Boldrini, J.L. And Rojas-Medar, M.A., On a system of evolution equations of Magnetohydrodinamic type, Mat. Cont., 8, 1-19, 1995.

[3] Constantin, P. and Foias C., Navier-Stokes equations, The Univ. Chicago Press, Chicago and London, 1988.

[4] Damásio, P. and Rojas-Medar, M.A., On some questions of the weak solutions of evolution equations for magnctohydrodynamic type, Proyecciones 16(1997), 83-97..

[5] Fujita, H. and Kato, T., On the Navier-Stokes initial value problem I, Arch. Rat. Mech. Anal. 16(1964), 269-315.

[6] Lassner, G., Über Ein Rand-Anfangswert - Problem der Magnetohydrodinamik, Arch. Rat. Mech. Anal. 25 (1967), 388-405.

[7] Lions, J.L., Quelques Methods de Resolution des Problémes aux Limits Non Linéares, Dunod, Paris, 1969.

[8] Pikelner, S.B., Grundlangen der Kosmischen Elektrohydrodynamik, Moscou, 1966.

[9] Rojas-Medar, M.A. and Beltrán-Barrios, R., The initial value problem for the equations of magnetohydrodynamic type in noncylindrical domains, Rev. Mat. Univ. Compl. Madrid, 8 (1995), 229-251. 
[10] Rojas-Medar, M.A. and Boldrini, J.L. Global strong solutions of equations of magnetohydrodynamic type, J. Australian Math. Soc., Serie B, Applied Math 38 (1997), 291-306.

[11] Schlüter, A., Dynamic des Plasmas, I and II. Z. Naturforsch. 5 a $(1950)$, $72-78 ; 6^{\mathrm{a}},(1951), 73-79$.

[12] Sedov, V.I. and Fokht, A.S., Correctness of Fitz Hugh's problem, Diff. Urav. 16 (1980), 114-1121.

[13] Temam, R., Navier-Stokes equations, North-Holland, Amsterdam, Rev. Edit., 1979.

[14] Zarubin, A.G., On an iterational method for the approximate solution of an initial - and boundary-value problem for the heat-convection equations, Comput. Math. Phys. 33 (1993), 1077-1085.

Received : April 10, 1998

\section{Eduardo Notte Cuello}

Departamento de Matemáticas

Universidad de Antofagasta

Casilla 170

Antofagasta

Chile

and

\section{Marko Rojas Medar}

DMA - Instituto de Matemáticas, Estatística e Ciencia da Computacao Universidad Estadual de Campinas

Caixa Postal 6065

Campinas 13081

Campinas - Sao Paulo

Brasil 